\title{
Computation of Fisher-Gale Equilibrium by Auction
}

\author{
Yurii Nesterov ${ }^{1,2} \cdot$ Vladimir Shikhman $^{3}$
}

Received: 17 September 2017 / Revised: 13 January 2018 / Accepted: 19 January 2018 /

Published online: 16 February 2018

(C) The Author(s) 2018, corrected publication June 2018

\begin{abstract}
We study the Fisher model of a competitive market from the algorithmic perspective. For that, the related convex optimization problem due to Gale and Eisenberg (Ann Math Stat 30(1):165-168, 1959) is used. The latter problem is known to yield a Fisher equilibrium under some structural assumptions on consumers' utilities, e.g., homogeneity of degree 1 , homotheticity. Our goal is to examine applicability of the convex optimization framework by departing from these traditional assumptions. We just assume the concavity of consumers' utility functions. For this case, we suggest a novel concept of Fisher-Gale equilibrium by using consumers' utility prices. The prices of utility transfer the utility of consumption bundle to a common numéraire. We develop a subgradient-type algorithm from Convex Analysis to compute a Fisher-Gale equilibrium via Gale's approach. In order to decentralize prices, we additionally implement the auction design, i.e., consumers settle and update their
\end{abstract}

The original version of this article was revised due to a retrospective Open Access order.

The research of Yurii Nesterov was supported by the Russian Science Foundation (No.17-11-01207). The research of Vladimir Shikhman was supported by a Grant "Chargé de recherches 98444" from the "Fonds de la Recherche Scientifique-FNRS, Belgique".

$\bowtie$ Yurii Nesterov

yurii.nesterov@uclouvain.be

Vladimir Shikhman

vladimir.shikhman@mathematik.tu-chemnitz.de

1 Center for Operations Research and Econometrics, Catholic University of Louvain, 1348 Louvain-la-Neuve, Belgium

2 National Research University Higher School of Economics, Moscow, Russia

3 Department of Mathematics, Technische Universität Chemnitz, 09126 Chemnitz, Germany 
individual prices and producers sell at the highest offer price. Our price adjustment is based on a tatonnement procedure, i.e., the prices change proportionally to consumers' individual excess supplies. Historical averages of consumption are shown to clear the market of goods. Our algorithm is justified by a global rate of convergence. In the worst case, the number of price updates needed to achieve an $\varepsilon$-tolerance is proportional to $\frac{1}{\varepsilon^{2}}$.

Keywords Fisher equilibrium - Computation of equilibrium · Price adjustment . Convex optimization - Subgradient methods - Decentralization of prices · Auction

Mathematics Subject Classification 91B50 - 91B26 · 90C25

\section{Introduction}

The concept of Fisher equilibrium for a competitive market dates back to 1891, see, e.g., [1]. Due to Fisher's model, consumers buy goods by spending given wealths in order to maximize their utility functions. There are fixed amounts of supplied goods available at the market. Fisher equilibrium comprises of optimal consumption bundles and equilibrium prices which clear the market of goods. Aiming at the efficient computation of Fisher equilibrium, a related convex optimization problem has been proposed in [2]. This so-called Gale's problem consists of maximizing an aggregated logarithmic utility function subject to market feasibility constraints. The feasibility constraints ensure that the aggregated consumption does not exceed the fixed amounts of supplied goods. The solutions of Gale's problem give equilibrium allocations for the Fisher market. Moreover, the Lagrange (or dual) multipliers for its feasibility constraints yield equilibrium prices. It is crucial to point out that the solutions of Gale's problem provide Fisher equilibrium mainly if the wealths are fully spent within the budget constraints. To guarantee the latter fact, some structural assumptions on the consumers' utility functions have been made in the literature. In [2], the case of linear utility functions for Fisher market has been considered. Later, the Gale's approach has been extended for concave and homogeneous utility functions of degree one in [3]. The convex optimization framework has been applied in [4] in order to handle homothetic and quasi-concave utilities. Recently in [5], the particular case of concave and nonhomogeneous utility functions in potential or logarithmic form has been successively studied.

The goal of the present paper is to examine the applicability of Gale's approach by departing from the structural assumptions on the consumers' utilities. In what follows, we just assume the concavity of consumers' utility functions. In the case of general concave utility functions, we cannot guarantee a full spending of wealths within the budget constraints. This is the main reason why under our concavity assumption the concepts of Fisher and Gale equilibrium may come apart. To explain this feature, we generalize both concepts of Fisher and Gale equilibrium by using the so-called utility prices attributed to consumers. They play the role of trade-offs between consumers' budget spending and utility maximization. Prices of utility allow to dynamically transfer the utility of a consumption bundle to a common numéraire. Using this transferable 
utility, we introduce a novel concept of Fisher-Gale equilibrium. Here, consumers maximize their revenues defined as a difference of transferred utilities and expenditures expressed in a numéraire (see Definition 2.3 for details). It turns out that Fisher and Gale equilibria can be viewed as Fisher-Gale equilibrium (see Theorem 2.1). In particular, for Fisher equilibrium the utility prices are inverse shadow prices (or Lagrange multipliers associated to budget constraints). For Gale equilibrium, the utility prices appear as ratios of wealths to achieved utilities. The latter gives rise to the efficient computation of a Fisher-Gale equilibrium by following the Gale's approach. We revise some previous attempts to solve the Gale's convex optimization problem known in the literature. Already in [6] the ellipsoid method has been applied for that. In [7], a polynomial time algorithm based on a primal-dual scheme has been proposed to tackle the Gale's problem. An interior-point method for Gale's problem is developed in [8]. For an algorithm based on the excess demand function, we refer to [9]. An auction-based algorithm for Fisher model has been suggested in [10]. A distributed algorithm via gradient descent for Fisher market with linear utility and spending constraint utilities has been suggested in [11]. In [12], a decentralized algorithm with the tatonnement price adjustment has been constructed using the indirect utility functions. We also mention [13] where a simultaneous ascending auction is used to construct a decentralized price adjustment. For comprehensive surveys on the computational issues of economic equilibria, see $[14,15]$.

In this paper we develop a subgradient-type algorithm to compute a Fisher-Gale equilibrium by Gale's approach. Its convergence properties are crucially based on Convex Analysis. The price adjustment corresponds to the quasi-monotone subgradient method for nonsmooth convex minimization, recently suggested in [16]. As objective function for the latter method, we take the total logarithmic revenue of the market. Equilibrium prices can be then characterized as its minimizers. By doing so, we independently rediscovered the framework recently proposed in [17]. In [17], the minimization of the total logarithmic revenue has been studied in the smooth setting by using gradient method. For that, the authors concentrate on Leontief utilities and complementary constant elasticity of substitution (CES) utilities which induce a smooth total logarithmic revenue. As to be expected for the usual gradient method, their analysis provides $\frac{1}{\varepsilon}$ rate of convergence for the $\varepsilon$-tolerance. In contrast to this study, we minimize the total logarithmic revenue in the nonsmooth setting assuming just the concavity of consumers' utility functions. In this general case, the total logarithmic revenue need not to be smooth, as already the example with linear additive utilities shows (see Example 3.1).

In order to decentralize prices, we additionally implement the auction design:

consumers settle and update their individual prices,

and producers sell at the highest offer price.

It is crucial for our approach that the introduction of the auction design preserves convexity of the total logarithmic revenue. Moreover, its convex subgradients w.r.t. a consumer's price become the individual excess supplies, which are easily observable. This is used by consumers to successively update prices by themselves rather than by relying on a central authority. Our price adjustment is based on a tatonnement procedure, i.e., the prices change proportionally to consumers' individual excess supplies. 
While our algorithm proceeds, the market clearance is achieved on average. The latter means that during the price adjustment supply meets demand statistically. In mathematical terms, average consumption bundles approach the solution of the Gale's (or adjoint) problem for the minimization of the total logarithmic revenue. Altogether, the sequence of highest offer prices, historical averages of consumption bundles and historical averages of utility prices generated by our algorithm, converge to the set of Fisher-Gale equilibria (see Theorem 4.1). Moreover, our algorithm is able to guarantee a convergence rate of this process. In the worst case, the number of price updates needed to achieve an $\varepsilon$-tolerance is proportional to $\frac{1}{\varepsilon^{2}}$. Note that this rate of convergence is optimal for nonsmooth convex minimization, cf. [18]. From the economic perspective, this result explains why competitive markets adjust in efficient way; moreover, it quantifies the worst-case efficiency. Note that relatively low accuracy of price adjustment processes usually suffices for the markets. Consequently, our complexity result of $\frac{1}{\varepsilon^{2}}$ is quite reasonable.

The article is organized as follows. In Sect. 2, we introduce and discuss the concept of Fisher-Gale equilibrium. In Sect. 3, we describe the decentralization of prices by the auction. We prove the convergence of our decentralized subgradient-type algorithm toward the set of Fisher-Gale equilibria in Sect. 4. "Appendix" is devoted to the mathematical justification of quasi-monotone subgradient schemes.

Notation Our notation is quite standard. We denote by $\mathbb{R}^{n}$ the space of $n$-dimensional column vectors $x=\left(x^{(1)}, \cdots, x^{(n)}\right)^{\mathrm{T}}$, and by $\mathbb{R}_{+}^{n}$ the set of all vectors with nonnegative components. $\mathbb{R}_{++}$stands for the set of positive real numbers. For $x$ and $y$ from $\mathbb{R}^{n}$, we introduce the standard scalar product and the Hadamard product

$$
\langle x, y\rangle=\sum_{i=1}^{n} x^{(i)} y^{(i)}, \quad x \circ y=\left(x^{(i)} y^{(i)}\right)_{i=1}^{n} \in \mathbb{R}^{n} .
$$

For vectors $p_{1}, \cdots, p_{I} \in \mathbb{R}^{n}$, we denote by $\max _{i=1, \cdots, I} p_{i} \in \mathbb{R}^{n}$ the vector with coordinates

$$
\left(\max _{i=1, \cdots, I} p_{i}\right)^{(j)}=\max _{i=1, \cdots, I} p_{i}^{(j)}, \quad j=1, \cdots, n .
$$

\section{Fisher-Gale Equilibrium}

Let us start with the classical concept of Fisher equilibrium, see, e.g., [1]. Consider a market with $I$ consumers, which are able to buy $n$ divisible goods. The $i$ th consumer has to decide on the consumption bundle $x_{i} \in X_{i}$, where the consumption set $X_{i} \subset \mathbb{R}_{+}^{n}$ is assumed to be nonempty, closed, and convex with $0 \in X_{i}$. Given a vector of prices $p \in \mathbb{R}_{+}^{n}$, the $i$ th consumer maximizes the concave utility function $u_{i}: \mathbb{R}_{+}^{n} \rightarrow \mathbb{R}$ with respect to the so-called budget constraint. The latter says that the acquired consumption bundle cannot cost more than the available wealth $w_{i} \in \mathbb{R}_{+}$of the $i$ th consumer. We assume that the utility function $u_{i}$ is positive on the topological interior of the consumption set $\operatorname{int}\left(X_{i}\right)$, i.e., $u_{i}\left(x_{i}\right)>0$ for all $x_{i} \in \operatorname{int}\left(X_{i}\right)$. On the production side of the market, there are $K$ producers. Each of them supplies fixed amounts of goods as 
given by the vectors $e_{k} \in \mathbb{R}_{+}^{n}, k=1, \cdots, K$. The aggregate supply of goods is thus $e \stackrel{\text { def }}{=} \sum_{k=1}^{K} e_{k} \in \mathbb{R}_{+}^{n}$. Finally, equilibrium prices ensure the market clearing condition, i.e., the aggregate consumption never exceeds the available amounts of supplied goods, and the markets of goods with positive prices are perfectly cleared.

Definition 2.1 (Fisher equilibrium [1]) The vector of prices and consumption bundles $\left(p^{*},\left(x_{i}^{*}\right)_{i=1}^{I}\right)$ is called Fisher equilibrium, if

(i) consumers maximize utilities w.r.t. budget constraints, i.e.,

$$
x_{i}^{*} \in \arg \max _{\substack{x_{i} \in X_{i} \\\left\langle p^{*}, x_{i}\right\rangle \leqslant w_{i}}} u_{i}\left(x_{i}\right), \quad i=1, \cdots, I ;
$$

(ii) the market clearing condition holds, i.e.,

$$
p^{*} \geqslant 0, \quad e-\sum_{i=1}^{I} x_{i}^{*} \geqslant 0, \quad\left\langle p^{*}, e-\sum_{i=1}^{I} x_{i}^{*}\right\rangle=0 .
$$

In order to compute Fisher equilibrium, the following convex optimization problem has been proposed in $[2,6]$ :

$$
\max _{\substack{x_{i} \in X_{i} \\ i=1, \cdots, I}} \sum_{i=1}^{I} w_{i} \ln u_{i}\left(x_{i}\right) \text { such that } \sum_{i=1}^{I} x_{i} \leqslant e .
$$

The objective function in (2.3) may be viewed as a socially aggregated utility, i.e., the sum of consumers' wealths assessed by logarithmic utility factors. The feasibility constraint in (2.3) means that the aggregate consumption never exceeds the available amounts of supplied goods. Market prices appear naturally as Lagrange multipliers for the feasibility constraint. Indeed, due to the duality of convex optimization, we obtain for (2.3):

$$
\begin{aligned}
& \max _{\substack{x_{i} \in X_{i} \\
i=1, \cdots, I}} \min _{p \geqslant 0} \sum_{i=1}^{I} w_{i} \ln u_{i}\left(x_{i}\right)+\left\langle p, e-\sum_{i=1}^{I} x_{i}\right\rangle \\
= & \min _{p \geqslant 0}\left\{\sum_{i=1}^{I} \max _{x_{i} \in X_{i}}\left[w_{i} \ln u_{i}\left(x_{i}\right)-\left\langle p, x_{i}\right\rangle\right]+\langle p, e\rangle\right\} .
\end{aligned}
$$

The latter saddle point problem can be interpreted economically as follows. Given the vector of prices $p \in \mathbb{R}_{+}^{n}$, the $i$ th consumer maximizes the logarithmic revenue by computing

$$
\operatorname{LR}_{i}(p) \stackrel{\text { def }}{=} \max _{x_{i} \in X_{i}}\left[w_{i} \ln u_{i}\left(x_{i}\right)-\left\langle p, x_{i}\right\rangle\right]
$$

Here, the logarithmic revenue is given as the difference between $i$ th consumer's logarithmically assessed wealth and his expenditures. Finally, the equilibrium prices are 
characterized by minimizing the total logarithmic revenue of consumers and producers:

$$
\operatorname{TLR}(p) \stackrel{\text { def }}{=} \sum_{i=1}^{I} \operatorname{LR}_{i}(p)+\langle p, e\rangle
$$

Motivated by the forgoing discussion, we define

Definition 2.2 (Gale equilibrium [2,6]) The vector of prices and consumption bundles $\left(p^{*},\left(x_{i}^{*}\right)_{i=1}^{I}\right)$ is called Gale equilibrium, if it solves the saddle point problem

$$
\min _{p \geqslant 0}\left\{\sum_{i=1}^{I} \max _{x_{i} \in X_{i}}\left[w_{i} \ln u_{i}\left(x_{i}\right)-\left\langle p, x_{i}\right\rangle\right]+\langle p, e\rangle\right\} .
$$

Namely,

(i) consumers maximize logarithmic revenues, i.e.,

$$
x_{i}^{*} \in \arg \max _{x_{i} \in X_{i}}\left[w_{i} \ln u_{i}\left(x_{i}\right)-\left\langle p^{*}, x_{i}\right\rangle\right], \quad i=1, \cdots, I
$$

(ii) the market clearing condition holds, i.e.,

$$
p^{*} \geqslant 0, \quad e-\sum_{i=1}^{I} x_{i}^{*} \geqslant 0, \quad\left\langle p^{*}, e-\sum_{i=1}^{I} x_{i}^{*}\right\rangle=0 .
$$

It is well known in the literature under which conditions the concepts of Fisher and Gale equilibrium coincide. In the case of $X_{i}=\mathbb{R}_{+}^{n}$ and linear utility functions $u_{i}(\cdot), i=1, \cdots, I$, the equivalence of Fisher and Gale equilibrium has served as a starting point for the seminal paper [2]. In [3], the equivalence result has been generalized for concave and homogeneous utility functions of degree 1 . The convex optimization framework (2.3) has been applied in [4] in order to handle homothetic and quasi-concave utilities. Recently in [5], the case of concave and nonhomogeneous utility functions in potential or logarithmic form has been successively tackled. It is worth to mention that the equivalence of Fisher and Gale concepts crucially relies on the full spending of wealths within the budget constraints. It turns out that the structural assumptions on the utilities provide the latter fact. The goal of the present paper is to examine the applicability of the convex optimization approach (2.3) by departing from the structural assumptions on the consumers' utilities. We merely state

Assumption 2.1 (Concavity) Utility functions $u_{i}(\cdot), i=1, \cdots, I$, are concave.

Note that Assumption 2.1 is standard in consumption theory, expressing the fact that consumers are risk averse, e.g., [19].

As a further novelty, we introduce general compact consumption sets $X_{i}$ rather than $X_{i}=\mathbb{R}_{+}^{n}$ as in the previous literature. If not stated otherwise, it holds throughout the paper: 
Assumption 2.2 (Compactness) Consumption sets $X_{i}, i=1, \cdots, I$, are compact.

Note that Assumption 2.2 refers to the fact that the needs of consumers are bounded. Since this is not standard, let us properly justify it from the economic point of view.

Remark 2.1 (Justification of Assumption 2.2) We start with the citation [20], where Assumption 2.2 has been discussed in the framework of jungle equilibrium. Michele Piccione and Ariel Rubinstein argue in [20]: "Some readers may object to the assumption that the consumption of agents is bounded. . . We disagree with these objections. First, we do not find the presence of bounds on consumption less genuine than the absence of such bounds. Naturally, there are physical limits to what people can consume." As it is pointed out in [21], one of these physical limits may be the time constraint. Andrew Schorrer argues in his textbook [21, Page 27] as follows: “... our consumption set is unbounded from above, which leads to the assumption that our agents can consume infinite positive amounts of the two goods available to them - an assumption that is clearly unreasonable. To make our analysis more realistic, we need only recognize that agents cannot consume infinite amounts of goods for a variety of reasons. For example, consumption takes time, and in any given day, there is not enough time to consume more than a finite amount of each good."

In [22], Mont summarizes the general critics on the neoclassical consumption theory: "Taking into account the concept of bounded rationality with lack of information and cognitive limitations, it is clear that consumers cannot be efficient in their choices and that neoclassical economics failed to provide sufficient explanation of consumption processes. Consumer behavior has been found to be far more complicated than just rational response to price signals being influenced by different internal and external drivers induced by human psychology, social norms and institutional settings. Since then, the neoclassical economic theory has been criticized by many scholars for its oversimplification of reality, particularly on the assumptions of a rational and sovereign consumers with limitless consumption needs." With respect to this critics, Assumption 2 just states that there are natural bounds on consumption needs which are modeled explicitly by compact consumption sets $X_{i}$. Note that the unbounded desire for wealth is not an issue here, since the wealth $w_{i}$ is a primitive in Fisher's model. Confer how Michele Piccione and Ariel Rubinstein comment on the wealth in [20]: “... we agree that it is not plausible to posit that agents have a bounded desire for wealth. However, we believe that it is not sensible to model the desire for ever increasing wealth in the same fashion as we model the desire to satisfy basic needs."

Let us finally discuss the mathematical aspects of Assumption 2.2. The latter guarantees that the consumers' demands from Definition 2.2

$$
\arg \max _{x_{i} \in X_{i}}\left[w_{i} \ln u_{i}\left(x_{i}\right)-\left\langle p, x_{i}\right\rangle\right]
$$

are uniformly bounded for all prices $p \in \mathbb{R}_{+}^{n}$. Actually, we need exactly this weaker property of consumers' demands for our analysis [cf. (4.31) from the proof of Lemma 4.1 in Appendix]. In the absence of Assumption 2.2, consumers' demands in (2.7) may be unbounded. For example, in case of $X_{i}=\mathbb{R}_{+}^{n}$ and monotone utility function $u_{i}$, consumers' demands (2.7) tend to infinity if prices $p$ go to zero. The same technical problem arises for standard consumer's demands from Definition 2.1: 


$$
\arg \max _{\substack{x_{i} \in X_{i} \\\left\langle p, x_{i}\right\rangle \leqslant w_{i}}} u_{i}\left(x_{i}\right) .
$$

In the context of general equilibrium theory, Thomas Marschak and Reinhard Selten suggest to resolve it in [23] as follows. "Turning to the case of unbounded consumption sets, $\cdots$ it is natural to attempt the procedure used by Debreu to deal with the difficulty posed by unboundedness, namely, that when certain prices are zero, consumer's . . choices might be infinite quantities. ... The procedure is to intersect each consumption set with a cube containing in its interior, the (bounded) set of all consumptions attainable if the economy's endowment and its technology were placed at the disposal of that consumer..." Assumption 2.2 can be achieved by using an analogous explicit construction. By doing so, the restriction $x_{i} \in X_{i}$ does not change the set of Gale or Fisher equilibria.

Note that in general if $X_{i}$ is sufficiently small and so the prices $p$ are, the restriction $x_{i} \in X_{i}$ can be binding in (2.7) and/or (2.8). Our framework allows those situations, as, e.g., people restrict their consumption of sweets although their preferences are usually assumed to be monotone. Let us illustrate this by considering the expenditure minimization problem

$$
e(p, u) \stackrel{\text { def }}{=} \min _{\substack{x \in X \\ u(x) \geqslant u}}\langle p, x\rangle,
$$

along with the utility maximization problem from (2.7):

$$
v(p, w) \stackrel{\text { def }}{=} \max _{\substack{x \in X \\\langle p, x\rangle \leqslant w}} u(x) .
$$

Here, we omit the index $i$ for simplicity. Denote by $h(p, u)$ and $x(p, w)$ the solution sets of (2.9) and (2.10), i.e., Walrasian and Hicksian demand correspondences, respectively. It is well known, e.g., [19] that assuming $X=\mathbb{R}_{+}^{n}$ and $u$ given by nonsatiated preferences, it holds:

$$
x(p, e(p, u))=h(p, u) \text { and } x(p, w)=h(p, v(p, w)) .
$$

For a compact consumption set $X$, i.e., under Assumption 2.2, the relation between (2.9) and (2.10) may be weaker than in (2.11). Namely, we merely have the existence of $\bar{x} \in h(p, u)$ such that $v(p, e(p, u))=u(\bar{x})$, as well as the existence of $\tilde{x} \in x(p, w)$ such that $e(p, v(p, w))=\langle p, \tilde{x}\rangle$. This is due to the fact that the budget constraint need not to be active. Nevertheless, if (2.9) and (2.10) are uniquely solvable, the usual relation (2.11) still holds even if $X$ is compact.

Overall, we emphasize that Assumption 2.2 has been successively used in the microeconomic literature, it is meaningful in our setting, and it is far from being restrictive.

In the case of general concave utility functions and compact consumption sets, we cannot guarantee the full spending of wealths within the budget constraints. This is the main reason why under our assumptions the concepts of Fisher and Gale equilibrium 
need not to coincide in general. To explain this feature, we generalize both concepts of Fisher and Gale equilibria by using the so-called utility prices $q_{i} \in(0, \infty]$ attributed to the $i$ th consumer. Prices of utility $q_{i}$ allow to dynamically transfer the utility $u_{i}\left(x_{i}\right)$ of a consumption bundle $x_{i}$ to a common numéraire by $q_{i} u_{i}\left(x_{i}\right)$. For the discussion on the concept of transferable utility, we refer, e.g., to [19].

Definition 2.3 (Fisher-Gale equilibrium) The vector of prices and consumption bundles $\left(p^{*},\left(x_{i}^{*}\right)_{i=1}^{I}\right)$ is called Fisher-Gale equilibrium w.r.t. utility prices $\left(q_{i}\right)_{i=1}^{I}$, if

(i) consumers maximize revenues fulfilling budget constraints, i.e.,

$$
x_{i}^{*} \in \arg \max _{x_{i} \in X_{i}}\left[q_{i} u_{i}\left(x_{i}\right)-\left\langle p^{*}, x_{i}\right\rangle\right], \text { and }\left\langle p^{*}, x_{i}^{*}\right\rangle \leqslant w_{i}, \quad i=1, \cdots, I
$$

(ii) the market clearing condition holds, i.e.,

$$
p^{*} \geqslant 0, \quad e-\sum_{i=1}^{I} x_{i}^{*} \geqslant 0, \quad\left\langle p^{*}, e-\sum_{i=1}^{I} x_{i}^{*}\right\rangle=0 .
$$

Note that the utility price $q_{i}=\infty$ in (2.12) means that $x_{i}^{*} \in \arg \max _{x_{i} \in X_{i}} u_{i}\left(x_{i}\right)$.

We define the concept of Fisher-Gale equilibrium in terms of not only the price vector, utility functions, and budgets, but also the utility prices. The utility prices can be fixed as characteristics of a consumer (see Example 2.1) or be adjusted dynamically by his behavioral patterns (see Theorem 2.1). In what follows, we discuss the novel concept of Fisher-Gale equilibrium in detail. First, note that utility prices $\left(q_{i}\right)_{i=1}^{I}$ from Definition 2.3 play the role of trade-offs between consumers' budget spending and utility maximization. By properly choosing utility prices, the consumers may keep some budget unspent. The latter will cause the sacrifices in their achieved utility. Next Example 2.1 highlights this issue. Here, we examine the consumer's revenue maximization (2.12) for homogeneous utility functions of degree $\gamma \in(0,1)$.

Example 2.1 (Homogeneity of degree $\gamma \in(0,1))$ Let us consider the consumer's revenue maximization as in (2.12):

$$
\max _{x \geqslant 0}[q u(x)-\langle p, x\rangle], \text { and }\langle p, x\rangle \leqslant w
$$

where the utility function $u$ is homogeneous of degree $\gamma \in(0,1)$, i.e.,

$$
u(t x)=t^{\gamma} u(x) \text { for all } x, t \geqslant 0 .
$$

Substituting $x=t y$ into (2.14), we have

$$
\max _{x \geqslant 0}[q u(x)-\langle p, x\rangle]=\max _{y, t \geqslant 0}\left[q t^{\gamma} u(y)-t\langle p, y\rangle\right]
$$


Maximizing first w.r.t. $t$ for a fixed $y$, we obtain

$$
t=\left[\frac{\gamma q u(y)}{\langle p, y\rangle}\right]^{\frac{1}{1-\gamma}} .
$$

Substituting this formula into (2.15), we get

$$
\max _{y \geqslant 0}\left[\frac{q u(y)}{\langle p, y\rangle^{\gamma}}\right]^{\frac{1}{1-\gamma}} \gamma^{\frac{\gamma}{1-\gamma}}(1-\gamma) .
$$

Due to the homogeneity of $u(\cdot)$ of degree $\gamma$, this maximization problem is equivalent to

$$
\max _{\substack{\langle p, y\rangle=1 \\ y \geqslant 0}} u(y) .
$$

Here, one unit of the numéraire is spent optimally w.r.t. the usual utility maximization. Having a solution $y^{*}$ of (2.16), we obtain a solution of (2.15):

$$
x^{*}=t y^{*}=\left[\gamma q u\left(y^{*}\right)\right]^{\frac{1}{1-\gamma}} y^{*} .
$$

The optimal budget spending and achieved utility are

$$
\left\langle p, x^{*}\right\rangle=\left[\gamma q u\left(y^{*}\right)\right]^{\frac{1}{1-\gamma}}, \quad u\left(x^{*}\right)=\left[\gamma q u\left(y^{*}\right)\right]^{\frac{\gamma}{1-\gamma}} u\left(y^{*}\right) .
$$

Further, note that from $\left\langle p, x^{*}\right\rangle \leqslant w$, we deduce that the utility price need to satisfy:

$$
q \leqslant \frac{w^{1-\gamma}}{\gamma u\left(y^{*}\right)}
$$

From here we see that utility prices compromise both the budget spending and the achieved utility. In particular, if $q \rightarrow 0$, then $\left\langle p, x^{*}\right\rangle \rightarrow 0$ and $u\left(x^{*}\right) \rightarrow 0$; if $q \rightarrow \frac{w^{1-\gamma}}{\gamma u\left(y^{*}\right)}$, then $\left\langle p, x^{*}\right\rangle \rightarrow w$ and $u\left(x^{*}\right) \rightarrow w^{\gamma} u\left(y^{*}\right)$.

Further, it turns out that by setting particular utility prices in (2.14) we recover Fisher's utility maximization (2.1) and Gale's logarithmic revenue maximization (2.5). Indeed, if $q=\frac{w^{1-\gamma}}{\gamma u\left(y^{*}\right)}$, then $\left\langle p, x^{*}\right\rangle=w$, and $x^{*}=w y^{*}$. Here, the whole budget is spent, and we have the optimal consumption of Fisher's utility maximization (2.1). If $q=\frac{w^{1-\gamma}}{\gamma^{\gamma} u\left(y^{*}\right)}$, then $\left\langle p, x^{*}\right\rangle=\gamma w$, and $x^{*}=\gamma w y^{*}$. The latter gives us the optimal consumption of Gale's logarithmic revenue maximization (2.5).

Next Theorem 2.1 shows in general that equilibria due to Fisher and Gale are particular cases of Fisher-Gale equilibrium. For Fisher equilibrium, the utility prices arise as inverse shadow prices (or Lagrange multipliers associated to budget constraints). For Gale equilibrium, the utility prices can be found as ratios of wealths to achieved utility values. 


\section{Theorem 2.1}

(a) If $\left(p^{*},\left(x_{i}^{*}\right)_{i=1}^{I}\right)$ is a Fisher equilibrium with Lagrange multipliers $\lambda_{i}^{*}$ associated to budget constraints in (2.1), then $\left(p^{*},\left(x_{i}^{*}\right)_{i=1}^{I}\right)$ is a Fisher-Gale equilibrium w.r.t. utility prices $\left(\frac{1}{\lambda_{i}^{*}}\right)_{i=1}^{I}$.

(b) If $\left(p^{*},\left(x_{i}^{*}\right)_{i=1}^{I}\right)$ is a Gale equilibrium, then $\left(p^{*},\left(x_{i}^{*}\right)_{i=1}^{I}\right)$ is a Fisher-Gale equilibrium w.r.t. utility prices $\left(\frac{w_{i}}{u_{i}\left(x_{i}^{*}\right)}\right)_{i=1}^{I}$.

Proof

(a) Let $\left(p^{*},\left(x_{i}^{*}\right)_{i=1}^{I}\right)$ be a Fisher equilibrium according to Definition 2.1. Optimality conditions for (2.1) read

$$
\begin{gathered}
\left\langle\nabla u_{i}\left(x_{i}^{*}\right)-\lambda_{i}^{*} p^{*}, x_{i}^{*}-y_{i}\right\rangle \geqslant 0 \text { for all } y_{i} \in X_{i}, \\
\lambda_{i}^{*} \geqslant 0,\left\langle p^{*}, x_{i}^{*}\right\rangle \leqslant w_{i}, \lambda_{i}^{*}\left(w_{i}-\left\langle p^{*}, x_{i}^{*}\right\rangle\right)=0,
\end{gathered}
$$

Due to concavity of utility functions $u_{i}(\cdot), i=1, \cdots, I$, we have

$$
\left\langle\nabla u_{i}\left(x_{i}^{*}\right), x_{i}^{*}-y_{i}\right\rangle \leqslant u_{i}\left(x_{i}^{*}\right)-u_{i}\left(y_{i}\right) \quad \text { for all } y_{i} \in X_{i} .
$$

Together with (2.17) we obtain

$$
u_{i}\left(x_{i}^{*}\right)-u_{i}\left(y_{i}\right)-\lambda_{i}^{*}\left\langle p^{*}, x_{i}^{*}-y_{i}\right\rangle \geqslant 0 \quad \text { for all } y_{i} \in X_{i},
$$

thus, if $\lambda_{i}^{*} \neq 0$,

$$
\frac{1}{\lambda_{i}^{*}} u_{i}\left(x_{i}^{*}\right)-\left\langle p^{*}, x_{i}^{*}\right\rangle \geqslant \frac{1}{\lambda_{i}^{*}} u_{i}\left(y_{i}\right)-\left\langle p^{*}, y_{i}\right\rangle \geqslant 0 \quad \text { for all } y_{i} \in X_{i} .
$$

If $\lambda_{i}^{*}=0$, then the utility price is formally set to $\frac{1}{\lambda_{i}^{*}}=\infty$, and

$$
u_{i}\left(x_{i}^{*}\right) \geqslant u_{i}\left(y_{i}\right) \text { for all } y_{i} \in X_{i} .
$$

(b) Let $\left(p^{*},\left(x_{i}^{*}\right)_{i=1}^{I}\right)$ be a Gale equilibrium according to Definition 2.2. Optimality conditions for (2.5) read

$$
\left\langle\frac{w_{i}}{u_{i}\left(x_{i}^{*}\right)} \nabla u_{i}\left(x_{i}^{*}\right)-p^{*}, x_{i}^{*}-y_{i}\right\rangle \geqslant 0 \quad \text { for all } y_{i} \in X_{i} .
$$

Again using (2.18), we obtain

$$
\frac{w_{i}}{u_{i}\left(x_{i}^{*}\right)} u_{i}\left(x_{i}^{*}\right)-\left\langle p^{*}, x_{i}^{*}\right\rangle \geqslant \frac{w_{i}}{u_{i}\left(x_{i}^{*}\right)} u_{i}\left(y_{i}\right)-\left\langle p^{*}, y_{i}\right\rangle \text { for all } y_{i} \in X_{i} .
$$


Moreover, setting $y_{i}=0$ in (2.19) and in (2.18), we have

$$
\left\langle p^{*}, x_{i}^{*}\right\rangle \leqslant w_{i} \frac{\left\langle\nabla u_{i}\left(x_{i}^{*}\right), x_{i}^{*}\right\rangle}{u_{i}\left(x_{i}^{*}\right)} \leqslant w_{i} \frac{u_{i}\left(x_{i}^{*}\right)-u_{i}(0)}{u_{i}\left(x_{i}^{*}\right)} \leqslant w_{i} .
$$

Overall, the assertions (a) and (b) follow.

Using Theorem 2.1, we relate the Fisher-Gale equilibrium to the well-known Negishi's approach to exchange equilibria from [24].

Remark 2.2 (Negishi's approach and Fisher-Gale equilibrium) In [24], Negishi characterizes exchange equilibria as welfare maximizers by appropriately choosing utility prices. In order to apply Negishi's approach, we equivalently reformulate Fisher equilibrium in terms of exchange. For that, we assign to every consumer a fraction of the producers' supplied goods proportional to his wealth. We call the vector of prices and consumption bundles $\left(p^{*},\left(x_{i}^{*}\right)_{i=1}^{I}\right)$ exchange equilibrium, if

(i) consumers maximize utilities w.r.t. modified budget constraints, i.e.,

$$
\begin{gathered}
x_{i}^{*} \in \arg \max _{x_{i} \in X_{i}} u_{i}\left(x_{i}\right), \quad i=1, \cdots, I ; \\
\left\langle p^{*}, x_{i}\right\rangle \leqslant\left\langle p^{*}, \frac{w_{i}}{\sum_{i=1}^{I} w_{i}} e\right\rangle ;
\end{gathered}
$$

(ii) the market clearing condition holds, i.e.,

$$
p^{*} \geqslant 0, \quad e-\sum_{i=1}^{I} x_{i}^{*} \geqslant 0, \quad\left\langle p^{*}, e-\sum_{i=1}^{I} x_{i}^{*}\right\rangle=0 .
$$

It is straightforward to see that the concepts of Fisher and exchange equilibria are equivalent. Namely, if $\left(p^{*},\left(x_{i}^{*}\right)_{i=1}^{I}\right)$ is a Fisher equilibrium, then it is also an exchange equilibrium. Vice versa, if $\left(p^{*},\left(x_{i}^{*}\right)_{i=1}^{I}\right)$ is an exchange equilibrium, then $\left(\frac{\sum_{i=1}^{I} w_{i}}{\left\langle p^{*}, e\right\rangle} p^{*},\left(x_{i}^{*}\right)_{i=1}^{I}\right)$ is a Fisher equilibrium. Here, we use the invariance of the exchange equilibrium under the scaling of prices.

The Negishi's welfare maximization is

$$
\max _{\substack{x_{i} \in X_{i} \\ i=1, \cdots I}}\left\{\sum_{i=1}^{I} q_{i} u_{i}\left(x_{i}\right) \mid \sum_{i=1}^{I} x_{i} \leqslant e\right\}
$$

where $q_{i}, i=1, \cdots, I$, are positive utility prices. Due to [24], if $\left(p^{*},\left(x_{i}^{*}\right)_{i=1}^{I}\right)$ is an exchange equilibrium, then there exist utility prices $\left(q_{i}\right)_{i=1}^{I}$ such that $\left(x_{i}^{*}\right)_{i=1}^{I}$ maximizes Negishi's welfare with dual (or Lagrange) multipliers $p^{*}$ w.r.t. the market feasibility constraints $\sum_{i=1}^{I} x_{i} \leqslant e$. As we have seen in Theorem 2.1(a), these 
utility prices can be taken as inverse shadow prices corresponding to the budget constraints. Also, the converse statement is given in [24]. Namely, fixing some utility prices in (2.20), its welfare maximizer with corresponding dual prices of goods forms an exchange equilibrium, but in general with redistributed wealths. Hence, in order to find a Fisher equilibrium, it is sufficient to determine utility prices for (2.20) such that initial budget constraints are fulfilled. However, this task is as challenging as to compute a Fisher equilibrium itself. For example, in [25] an adjustment of utility prices according to the consumers' savings is studied. In our approach, we relax the concept of Fisher equilibrium by imposing budget constraints for Negishi's welfare maximizers:

$$
\begin{gathered}
\left(x_{i}^{*}\right)_{i=1}^{I} \in \arg \max _{\substack{x_{i} \in X_{i} \\
i=1, \cdots I}}\left\{\sum_{i=1}^{I} q_{i} u_{i}\left(x_{i}\right) \mid \sum_{i=1}^{I} x_{i} \leqslant e\right\}, \\
\text { and }\left\langle p^{*}, x_{i}^{*}\right\rangle \leqslant w_{i}, \quad i=1, \cdots, I,
\end{gathered}
$$

where prices $p^{*}$ are dual multipliers w.r.t. the market feasibility. After a moment of reflection, we see that $\left(p^{*},\left(x_{i}^{*}\right)_{i=1}^{I}\right)$ from (2.21) is a Fisher-Gale equilibrium.

Theorem 2.1 clarifies how consumers may settle utility prices in a meaningful way, i.e., in consistency with their economic behavior. At least two possibilities are

- inverse shadow prices,

- wealth/utility ratios.

In this paper, we examine the adjustment of utility prices according to the wealth/utility relation by following the Gale's approach. For that, we assume that the $i$ th consumer is able to compute an optimal consumption bundle $x_{i}$ by maximizing the logarithmic revenue (2.4), i.e.,

$$
x_{i} \in \arg \max _{x_{i} \in X_{i}}\left[w_{i} \ln u_{i}\left(x_{i}\right)-\left\langle p, x_{i}\right\rangle\right]
$$

for a fixed vector of prices $p \in \mathbb{R}_{+}^{n}$. Let us provide algorithmic and economic justifications for this assumption.

(1) Algorithmic justification for logarithmic revenue maximization

As we have seen in the proof of Theorem 2.1(b), a consumption bundle $x_{i}$ from (2.22)

- satisfies the budget constraint, i.e., $\left\langle p, x_{i}\right\rangle \leqslant w_{i}$;

- maximizes revenue with the utility price $q_{i}=\frac{w_{i}}{u_{i}\left(x_{i}\right)}$, i.e.,

$$
q_{i} u_{i}\left(x_{i}\right)-\left\langle p, x_{i}\right\rangle \geqslant q_{i} u_{i}\left(y_{i}\right)-\left\langle p, y_{i}\right\rangle, \quad \text { for all } y_{i} \in X_{i} \text {. }
$$

In accordance with this interpretation, we always associate the utility price $q_{i}=\frac{w_{i}}{u_{i}\left(x_{i}\right)}$ with the consumption bundle $x_{i}$. The maximization of the logarithmic revenue as in (2.22) can be performed unintentionally by subgradient dynamics

$$
\dot{x}_{i} \in \frac{w}{u_{i}(x)} \nabla u_{i}(x)-p .
$$


In order to form the subgradient $\frac{w}{u_{i}(x)} \nabla u_{i}(x)-p$ of the logarithmic revenue, the consumer estimates the marginal utility $\nabla u_{i}(x)$ which is further reassessed by the utility price $\frac{w}{u_{i}(x)}$. Here, the utility price is taken as the wealth/utility ratio. Finally, the comparison of this reassessed marginal utility $\frac{w}{u_{i}(x)} \nabla u_{i}(x)$ with the prices of goods $p$ is performed. Thus, it is reasonable to assume that $\frac{w}{u_{i}(x)} \nabla u_{i}(x)-p$ can be used in (2.24) by the $i$ th consumer. Note that there is evidence from behavioral economics that consumer's choices need not be consistent with the maximization of a preference relation (see [26] and references therein). The reason for that is usually referred to as consumers' bounded rationality. Classic examples include status quo biases, attraction, compromise and framing effects, temptation and self-control, consideration sets, and choice overload. Within our approach, the consumption based on the maximization of the logarithmic revenue is consistent with the concept of transferable utility (cf. also [27]). Further, we mention that the discretization of (2.24) leads to subgradient schemes for nonsmooth convex optimization. Those are known to enjoy guaranteed rates of convergence [18]. This explains how consumers efficiently maximize the logarithmic revenue by successively using its subgradients $\frac{w}{u_{i}(x)} \nabla u_{i}(x)-p$.

(2) Economic justification for logarithmic revenue maximization

Let us define a quasilinear utility function for the $i$ th consumer as follows:

$$
U\left(x_{i}, \tau_{i}\right) \stackrel{\text { def }}{=} w_{i} \ln u_{i}\left(x_{i}\right)+\tau_{i}
$$

where $\tau_{i} \in \mathbb{R}_{+}$denotes the unspent numéraire. We consider the $i$ th consumer's quasilinear utility maximization [19]:

$$
\max _{\substack{\left\langle p, x_{i}\right\rangle+\tau_{i} \leqslant w_{i} \\ x_{i} \in X_{i}, \tau_{i} \geqslant 0}} U_{i}\left(x_{i}, \tau_{i}\right) .
$$

Due to (2.25), the budget constraint $\left\langle p, x_{i}\right\rangle+\tau_{i} \leqslant w_{i}$ is tight for optimal consumption bundles $x_{i}$, and we get

$$
\begin{aligned}
\max _{\substack{\left\langle p, x_{i}\right\rangle+\tau_{i} \leqslant w_{i} \\
x_{i} \in X_{i}, \tau_{i} \geqslant 0}} U_{i}\left(x_{i}, \tau_{i}\right)= & \max _{\substack{\left\langle p, x_{i}\right\rangle+\tau_{i}=w_{i} \\
x_{i} \in X_{i}, \tau_{i} \geqslant 0}}\left[w_{i} \ln u_{i}\left(x_{i}\right)+\tau_{i}\right] \\
= & w_{i}+\max _{\substack{\left\langle p, x_{i}\right\rangle \leqslant w_{i} \\
x_{i} \in X_{i}}}\left[w_{i} \ln u_{i}\left(x_{i}\right)-\left\langle p, x_{i}\right\rangle\right] \\
= & w_{i}+\max _{x_{i} \in X_{i}}\left[w_{i} \ln u_{i}\left(x_{i}\right)-\left\langle p, x_{i}\right\rangle\right] .
\end{aligned}
$$

The latter equality follows from the proof of Theorem $2.1(\mathrm{~b})$, since $\left\langle p, x_{i}\right\rangle \leqslant w_{i}$ always holds for optimal consumption bundles of the logarithmic revenue maximization. Thus, the constraint $\left\langle p, x_{i}\right\rangle \leqslant w_{i}$ is superfluous here. In case of a general quasilinear utility, this budget constraint is usually neglected by an assumption of no income effects [19]. No income effects mean that the available wealth $w_{i}$ does not affect consumption. This assumption has been questioned in the framework of Marshallian partial equilibrium analysis in [28,29]. In [29], the setting of a variable number of 
commodities is suggested. Sufficient conditions for a neoclassical utility function to induce small income effects are provided if the number of commodities is sufficiently large. In [28], a special class of quasilinear functions with restrictions for large income levels is studied. Due to our approach, an assumption on income effects is not needed. Overall, the logarithmic revenue maximization (2.22) is equivalent to the maximization of the particular quasilinear utility function (2.25) with respect to the budget constraint.

Finally, we compare the optimal budget spending for Fisher and Gale equilibria. In case of Fisher equilibrium, consumers face the full budget spending under standard monotonicity assumptions. In turn, for Gale equilibrium, the optimal budget spending is adjustable and depends on elasticities of utility functions.

Remark 2.3 (Budget spending) Assuming $X_{i}=\mathbb{R}_{+}^{n}$, we consider the Fisher's utility maximization

$$
\max _{\substack{x_{i} \geqslant 0 \\\left\langle p, x_{i}\right\rangle \leqslant w_{i}}} u_{i}\left(x_{i}\right) .
$$

If $u_{i}(\cdot)$ is strictly monotone, then the optimal budget spending $\left\langle p, x_{i}\right\rangle$ amounts to the available wealth $w_{i}$. In this context, the budget spending is fixed at the wealth level $w_{i}$ which should be known a priori as the amount of numéraire surely spent on the market under consideration. Moreover, every strictly monotone transformation of $u_{i}(\cdot)$ also induces the full budget spending (cf. Example 2.1). Hence, the optimal budget spending is not affected by the elasticities of the utility function $u_{i}$ [19]:

$$
\varepsilon_{j}\left(x_{i}\right) \stackrel{\text { def }}{=} \frac{\partial_{x_{j}} u_{i}\left(x_{i}\right)}{u_{i}\left(x_{i}\right)} \cdot x^{(j)}, \quad j=1, \cdots, n .
$$

In case of Gale's logarithmic revenue maximization

$$
\max _{x_{i} \geqslant 0}\left[w_{i} \ln u_{i}\left(x_{i}\right)-\left\langle p, x_{i}\right\rangle\right]
$$

the situation is different. In fact, assuming $u_{i}(\cdot)$ to be differentiable, we have necessary optimality conditions

$$
\frac{w}{u\left(x_{i}\right)} \nabla u\left(x_{i}\right)=p-s, \quad\left\langle s_{i}, x_{i}\right\rangle=0
$$

for an optimal consumption bundle $x_{i} \in \mathbb{R}_{+}^{n}$ and the vector of optimal dual multipliers $s_{i} \in \mathbb{R}_{+}^{n}$. Multiplying by $x_{i}$, we get

$$
\left\langle p, x_{i}\right\rangle=w_{i} \frac{\left\langle\nabla u\left(x_{i}\right), x_{i}\right\rangle}{u_{i}\left(x_{i}\right)}=w_{i} \sum_{j=1}^{n} \varepsilon_{j}\left(x_{i}\right) .
$$

This formula says that the ratio of the optimal budget spending $\left\langle p, x_{i}\right\rangle$ to the available wealth $w_{i}$ is the sum of elasticities $\varepsilon_{j}\left(x_{i}\right), j=1, \cdots, n$. Here, the optimal budget 
spending is adjusted depending on utility elasticities and is not known a priori. The available wealth $w_{i}$ has a role of its upper bound which may or may not be reached. Recall from the proof of Theorem 2.1(b) that

$$
\sum_{j=1}^{n} \varepsilon_{j}\left(x_{i}\right) \leqslant 1
$$

for a concave utility function $u_{i}$.

Finally, we describe the producers' behavior. Given individual consumers' prices $p_{1}, \cdots, p_{I} \in \mathbb{R}_{+}^{n}$, the $k$ th producer solves the distribution problem:

$$
\max _{\sum_{i=1}^{I} e_{i k}=e_{k}} \sum_{i=1}^{I}\left\langle p_{i}, e_{i k}\right\rangle=\left\langle\max _{i=1, \cdots, I} p_{i}, e_{k}\right\rangle .
$$

This means that $k$ th producer distributes resources $e_{k}$ among consumers with highest offer prices

$$
\max _{i=1, \cdots, I} p_{i}^{(j)}=\left(\max _{i=1, \cdots, I} p_{i}\right)^{(j)}, \quad j=1, \cdots, n
$$

Indeed, optimal solutions of (2.26) are distributions

$$
e_{i k}=\mu_{i k} \circ e_{k}, \quad\left(\mu_{i k}\right)_{i=1}^{I} \in M\left(p_{1}, \cdots, p_{I}\right)
$$

where

$$
M\left(p_{1}, \cdots, p_{I}\right) \stackrel{\text { def }}{=}\left\{\begin{array}{c|c}
\left(\mu_{i}\right)_{i=1}^{I} \in[0,1]^{n \times I} & \sum_{i=1}^{I} \mu_{i}^{(j)}=1 \\
\mu_{i}^{(j)}=0 \text { if } p_{i}^{(j)} \neq \max _{i=1, \cdots, I} p_{i}^{(j)} \\
j=1, \cdots, n, i=1, \cdots, I
\end{array}\right\} .
$$

Note that $\mu_{i k}^{(j)}$ denotes the share of $k$ th producer's supply $e_{k}$ to $i$ th consumer for good $j$. Indeed, the shares $\mu_{i k}^{(j)}$ for good $j$ sum up to 1 over all consumers $i=1, \cdots, I$. Moreover, the share $\mu_{i k}^{(j)}$ vanishes if the $i$ th consumer's price $p_{i}^{(j)}$ is less than the highest offer price $\max _{i=1, \cdots, I} p_{i}^{(j)}$ for good $j$. 


\section{Auction Design}

Theorem 2.1(b) suggests that for finding a Fisher-Gale equilibrium we may solve the following saddle point problem:

$$
\min _{p \geqslant 0}\left\{\sum_{i=1}^{I} \max _{x_{i} \in X_{i}}\left[w_{i} \ln u_{i}\left(x_{i}\right)-\left\langle p, x_{i}\right\rangle\right]+\langle p, e\rangle\right\} .
$$

First, we concentrate on the Fisher-Gale equilibrium prices as minimizers of the total logarithmic revenue, $\mathrm{cf}$. [17]:

$$
\mathrm{TLR}^{*} \stackrel{\text { def }}{=} \min _{p \in \mathbb{R}_{+}^{n}} \operatorname{TLR}(p)
$$

where

$$
\operatorname{TLR}(p)=\sum_{i=1}^{I} \operatorname{LR}_{i}(p)+\langle p, e\rangle, \quad \operatorname{LR}_{i}(p)=\max _{x_{i} \in X_{i}}\left[w_{i} \ln u_{i}\left(x_{i}\right)-\left\langle p, x_{i}\right\rangle\right]
$$

In order to ensure solvability in $(\mathrm{P})$, we assume that the market is productive. The productivity of the market means that there exist $\bar{x}_{i} \in X_{i}$ with $u_{i}(\bar{x})>0, i=1, \cdots, I$, such that the supply of goods strictly exceeds the aggregate demand, i.e.,

$$
\sum_{i=1}^{I} \bar{x}_{i}<e
$$

Actually, the market productivity can be viewed as a standard Slater condition for the logarithmic welfare maximization (2.3). It is well known that Slater condition implies the existence and boundedness of Lagrange multipliers (e.g., [30]), which are equilibrium prices in our context. Hence, at productive markets the set of equilibrium prices (or, equivalently, minimizers of the total logarithmic revenue TLR) is nonempty and bounded. From now on, let us assume the market productivity throughout.

As the maximum of linear functions, the total logarithmic revenue $\operatorname{TLR}(p)$ is convex w.r.t. the price $p$. However, the total logarithmic revenue is in general nonsmooth, even in case of homogeneous utilities. We illustrate this by examining markets with Leontief, Cobb-Douglas and linear additive utilities.

Example 3.1 (Leontief, Cobb-Douglas and linear additive utilities, cf. [17])

(a) Let consumers apply Leontief utility functions

$$
u_{i}\left(x_{i}\right)=\min _{1 \leqslant j \leqslant n} \frac{x_{i}^{(j)}}{b_{i}^{(j)}}
$$


where $b_{i}^{(j)}$ are positive scaling coefficients. This case corresponds to complementary goods. Assuming $X_{i}=\mathbb{R}_{+}$, we obtain after simple computations:

$$
\begin{aligned}
\operatorname{LR}_{i}(p) & =\max _{x_{i} \geqslant 0}\left[w_{i} \ln \left(\min _{1 \leqslant j \leqslant n} \frac{x_{i}^{(j)}}{b_{i}^{(j)}}\right)-\left\langle p, x_{i}\right\rangle\right] \\
& =-w_{i} \ln \left\langle p, b_{i}\right\rangle+w_{i}\left(\ln w_{i}-1\right),
\end{aligned}
$$

and, thus,

$$
\operatorname{TLR}(p)=-\sum_{i=1}^{I} w_{i} \ln \left\langle p, b_{i}\right\rangle+\langle p, e\rangle+\sum_{i=1}^{I} w_{i}\left(\ln w_{i}-1\right)
$$

In case of Leontief utilities, the total logarithmic revenue turns out to be smooth.

(b) Let consumers apply Cobb-Douglas utility functions

$$
u_{i}\left(x_{i}\right)=\alpha \prod_{j=1}^{n}\left(x_{i}^{(j)}\right)^{\alpha_{i}^{j}}
$$

where $\alpha_{i}^{j}$ are positive elasticities with $\sum_{j=1}^{n} \alpha_{i}^{j}=1$, and $\alpha$ is a positive scaling coefficient. This case also corresponds to complementary goods. Assuming $X_{i}=$ $\mathbb{R}_{+}$, we obtain after simple computations:

$$
\begin{aligned}
\operatorname{LR}_{i}(p) & =\max _{x_{i} \geqslant}\left[w_{i} \ln \left(\alpha \prod_{j=1}^{n}\left(x_{i}^{(j)}\right)^{\alpha_{i}^{j}}\right)-\left\langle p, x_{i}\right\rangle\right] \\
& =-w_{i} \sum_{j=1}^{n} \alpha_{i}^{j} \ln p^{(j)}+w_{i}\left(\ln \left(\alpha w_{i}\right)+\sum_{j=1}^{n} \alpha_{i}^{j} \ln \alpha_{i}^{j}-1\right)
\end{aligned}
$$

and, thus,

$$
\begin{aligned}
\operatorname{TLR}(p)= & -\sum_{i=1}^{I} w_{i} \sum_{j=1}^{n} \alpha_{i}^{j} \ln p^{(j)}+\langle p, e\rangle \\
& +\sum_{i=1}^{I} w_{i}\left(\ln \left(\alpha w_{i}\right)+\sum_{j=1}^{n} \alpha_{i}^{j} \ln \alpha_{i}^{j}-1\right) .
\end{aligned}
$$

In case of Cobb-Douglas utilities, the total logarithmic revenue turns out to be also smooth.

(c) Let consumers apply linear additive utility functions

$$
u_{i}\left(x_{i}\right)=\left\langle a_{i}, x_{i}\right\rangle=\sum_{j=1}^{n} a_{i}^{(j)} x_{i}^{(j)}
$$


where $a_{i}^{(j)}$ are positive scaling coefficients. This case corresponds to substitutionary goods. Assuming $X_{i}=\mathbb{R}_{+}$, we obtain after simple computations:

$\mathrm{LR}_{i}(p)=\max _{x_{i} \geqslant 0}\left[w_{i} \ln \left\langle a_{i}, x_{i}\right\rangle-\left\langle p, x_{i}\right\rangle\right]=-w_{i} \ln \left(\min _{1 \leqslant j \leqslant n} \frac{p^{(j)}}{a_{i}^{(j)}}\right)+w_{i}\left(\ln w_{i}-1\right)$,

and, thus,

$$
\operatorname{TLR}(p)=-\sum_{i=1}^{I} w_{i} \ln \left(\min _{1 \leqslant j \leqslant n} \frac{p^{(j)}}{a_{i}^{(j)}}\right)+\langle p, e\rangle+\sum_{i=1}^{I} w_{i}\left(\ln w_{i}-1\right) .
$$

In case of linear additive utilities, the total logarithmic revenue is nonsmooth. Hence, we emphasize that the total logarithmic revenue need not to be smooth. Also note that in all cases (a), (b) and (c) Gale equilibrium coincides with Fisher equilibrium, since Leontief, Cobb-Douglas and linear additive utilities are monotone and homogeneous.

Note that in [17] the minimization of the total logarithmic revenue has been studied in the smooth setting by assuming Leontief utilities and complementary constant elasticity of substitution (CES) utilities, such as Cobb-Douglas utilities. We present a nonsmooth treatment for the case of general concave utility functions. Our goal is to explain how agents can efficiently tackle the nonsmooth convex minimization problem (P) by successively updating prices. It is crucial for our approach that the updates of prices correspond to subgradient-type schemes for solving (P).

Theorem 3.1 (Subdifferential of TLR, cf. [17]) For $p \in \mathbb{R}_{+}^{n}$ it holds:

$$
\partial \operatorname{TLR}(p)=e-\sum_{i=1}^{I} \arg \max _{x_{i} \in X_{i}}\left[w_{i} \ln u_{i}\left(x_{i}\right)-\left\langle p, x_{i}\right\rangle\right] .
$$

Proof We apply [30, Theorem 23.8] on the subdifferential of the sum of convex functions in order to obtain

$$
\operatorname{\partial TLR}(p)=e-\sum_{i=1}^{I} \partial \operatorname{LR}_{i}(p)
$$

Due to [31, Theorem 2.4.18] on the convex subdifferential of a max-type function, we also have

$$
\partial \operatorname{LR}_{i}(p)=-\arg \max _{x_{i} \in X_{i}}\left[w_{i} \ln u_{i}\left(x_{i}\right)-\left\langle p, x_{i}\right\rangle\right], \quad i=1, \cdots, I .
$$

Overall, the assertion follows. 
Due to Theorem 3.1, the subgradients of TLR represent the excess supply, i.e.,

$$
\nabla \operatorname{TLR}(p)=e-\sum_{i=1}^{I} x_{i} \in \partial \operatorname{TLR}(p)
$$

where $x_{i} \in \arg \max _{x_{i} \in X_{i}} w_{i} \ln u_{i}\left(x_{i}\right)-\left\langle p, x_{i}\right\rangle$. This gives rise to use the subgradients $\operatorname{VTLR}(p)$ for the iterative minimization of TLR. For example, the change of prices $\Delta p$ can be taken proportional to the current excess demand:

$$
\Delta p \sim-\nabla \operatorname{TLR}(p)
$$

However, as it can be seen from (3.1), the subgradients of TLR are known neither to consumers nor to producers. Indeed, $\nabla \operatorname{TLR}(p)$ represents the aggregate excess supply. For getting access to its value, one would assume the existence of a manager who collects the information about all consumption bundles $x_{i}$, producers' fixed supplies $e_{k}$ and aggregates them over the whole market. Recall that $e=\sum_{k=1}^{K} e_{k}$. Here, the full information about consumption and production over the market must be available to the manager. Besides, the prices need to be updated by the manager, thus, leading to price regulation. Clearly, these assumptions can be justified only within a centrally planned economy. Aiming to avoid this restriction, we decentralize prices.

The decentralization of prices can be implemented by the introduction of the auction design:

$i$-th consumer settles and updates his individual prices $p_{i}$,

and producers sell at the highest offer price $\max _{i=1, \cdots, I} p_{i}$.

Note that for vectors $p_{1}, \cdots, p_{I} \in \mathbb{R}^{n}$, we denote by $\max _{i=1, \cdots, I} p_{i} \in \mathbb{R}^{n}$ the vector with coordinates

$$
\left(\max _{i=1, \cdots, I} p_{i}\right)^{(j)}=\max _{i=1, \cdots, I} p_{i}^{(j)}, \quad j=1, \cdots, n .
$$

Now, the total logarithmic revenue depends on the consumers' prices $\left(p_{i}\right)_{i=1}^{I}$ as follows:

$$
\begin{aligned}
\operatorname{TLR}\left(p_{1}, \cdots, p_{I}\right) & \stackrel{\text { def }}{=} \sum_{i=1}^{I} \operatorname{LR}_{i}\left(p_{i}\right)+\left\langle\max _{i=1, \cdots, I} p_{i}, e\right\rangle \\
& =\sum_{i=1}^{I} \max _{x_{i} \in X_{i}}\left[w_{i} \ln u_{i}\left(x_{i}\right)-\left\langle p_{i}, x_{i}\right\rangle\right]+\sum_{k}^{K}\left\langle\max _{i=1, \cdots, I} p_{i}, e_{k}\right\rangle
\end{aligned}
$$

The decentralization of prices makes the corresponding subdifferential information about excess demands available to consumers. In fact, note that the total logarithmic revenue TLR from (3.2) is convex in the variables $\left(p_{i}\right)_{i=1}^{I}$. Let us obtain an expression 
for its convex subgradients $\nabla_{p_{i}} \operatorname{TLR}\left(p_{1}, \cdots, p_{I}\right)$ w.r.t. $p_{i}$ :

$$
\nabla_{p_{i}} \operatorname{TLR}\left(p_{1}, \cdots, p_{I}\right)=\sum_{k}^{K} \mu_{i k} \circ e_{k}-x_{i}, \quad i=1, \cdots, I .
$$

Here, $x_{i} \in \arg \max _{x_{i} \in X_{i}}\left[w_{i} \ln u_{i}\left(x_{i}\right)-\left\langle p_{i}, x_{i}\right\rangle\right]$ is the demand of $i$ th consumer w.r.t. his individual price $p_{i}$. Further, with shares $\left(\mu_{i k}\right)_{i=1}^{I} \in M\left(p_{1}, \cdots, p_{I}\right)$ and due to (2.27),

$$
\left(\mu_{i k} \circ e_{k}\right)_{i=1}^{I}=\left(e_{i k}\right)_{i=1}^{I} \in \arg \max _{\sum_{i=1}^{I} e_{i k}} \sum_{i=1}^{I}\left\langle p_{i}, e_{i k}\right\rangle
$$

is an optimal resource distribution of $k$ th producer who faces consumers' prices $p_{1}, \cdots, p_{I}$.

We claim that the subdifferential information in (3.3) is known to $i$ th consumer. First, note that $x_{i}$ is his consumption bundle. Despite of the fact that the shares $\mu_{i k}$ and the supplies $e_{k}$ cannot be estimated by $i$ th consumer, their aggregate product $\sum_{k}^{K} \mu_{i k} \circ e_{k}$ is perfectly available to him. Indeed, $\sum_{k}^{K} \mu_{i k} \circ e_{k}$ forms the bundle of goods supplied by all producers to $i$ th consumer independently from each other. Altogether, the subgradients $\nabla_{p_{i}} \operatorname{TLR}\left(p_{1}, \cdots, p_{I}\right)$ represent the individual excess of $i$ th consumer's supply over his demands. Overall, we obtain:

Theorem 3.2 (Producers' excess supply and TLR)

$$
\begin{aligned}
& \partial_{p_{i}} \operatorname{TLR}\left(p_{1}, \cdots, p_{I}\right) \\
= & \sum_{k}^{K} \mu_{i k} \circ e_{k}-\arg \max _{x_{i} \in X_{i}}\left[w_{i} \ln u_{i}\left(x_{i}\right)-\left\langle p_{i}, x_{i}\right\rangle\right], \quad i=1, \cdots, I,
\end{aligned}
$$

with demand shares $\left(\mu_{i k}\right)_{i=1}^{I} \in M\left(p_{1}, \cdots, p_{I}\right)$.

Due to Theorem 3.2, the subdifferential of $\operatorname{TLR}\left(p_{1}, \cdots, p_{I}\right)$ is completely available to $i$ th consumer. This fact suggests to adjust prices by solving the minimization problem

$$
\min _{p_{1}, \cdots, p_{I} \in \mathbb{R}_{+}^{n}} \operatorname{TLR}\left(p_{1}, \cdots, p_{I}\right) .
$$

Note that the minimization problem (PD) is stated w.r.t. the decentralized consumers' prices $\left(p_{i}\right)_{i=1}^{I}$, while previously in $(\mathrm{P})$ one minimizes over the common prices $p$.

We relate the minimization problems (P) and (PD) by exploiting the fact that they have the same adjoint problem (2.3): 


$$
\max _{\substack{x_{i} \in X_{i} \\ i=1, \cdots, I}}\left\{\Phi\left(x_{1}, \cdots, x_{I}\right) \mid \sum_{i=1}^{I} x_{i} \leqslant e\right\}
$$

where

$$
\Phi\left(x_{1}, \cdots, x_{I}\right) \stackrel{\text { def }}{=} \sum_{i=1}^{I} w_{i} \ln u_{i}\left(x_{i}\right) .
$$

In (A) the central authority assigns consumption bundles by maximizing the logarithmic welfare of the society and by ensuring the market feasibility. In order to state (A), the central authority needs to know agents' utility functions, consumption sets, etc. Obviously, this information about the consumers is hardly observable to the central authority. Consequently, it cannot be justified in general that the welfare maximization problem is tackled directly. Nevertheless, note that the prices of goods play the role of Lagrange or dual multipliers for the market feasibility constraint

$$
\sum_{i=1}^{I} x_{i} \leqslant e
$$

Confer already $[6,32]$ for similar interpretations.

In order to prove that $(\mathrm{A})$ is the adjoint problem not only for $(\mathrm{P})$, but also for (PD), we need the following simple Lemma 3.1.

Lemma 3.1 For $x_{i}, e \in \mathbb{R}_{+}^{n}, i=1, \cdots, I$, the inequality

$$
\sum_{i=1}^{I} x_{i} \leqslant e
$$

is equivalent to

$$
\sum_{i=1}^{I}\left\langle p_{i}, x_{i}\right\rangle \leqslant\left\langle\max _{i=1, \cdots, I} p_{i}, e\right\rangle \text { for all } p_{i} \in \mathbb{R}_{+}^{n}, i=1, \cdots, I .
$$

Proof (i) Let (3.5) be satisfied. For $p_{i} \in \mathbb{R}_{+}^{n}, i=1, \cdots, I$, we have

$$
\sum_{i=1}^{I}\left\langle p_{i}, x_{i}\right\rangle-\left\langle\max _{i=1, \cdots, I} p_{i}, e\right\rangle=\sum_{j=1}^{n}\left(\sum_{i=1}^{I} p_{i}^{(j)} x_{i}^{(j)}-\max _{i=1, \cdots, I} p_{i}^{(j)} e^{(j)}\right) .
$$

For (3.6) to hold, it is sufficient to show that

$$
\sum_{i=1}^{I} p_{i}^{(j)} x_{i}^{(j)}-\max _{i=1, \cdots, I} p_{i}^{(j)} e^{(j)} \leqslant 0 \text { for all } j=1, \cdots, n .
$$


Indeed, setting for fixed $j \in\{1, \cdots, n\}$,

$$
p^{(j)}=\max _{i=1, \cdots, I} p_{i}^{(j)} \text { and } \mathcal{I}^{(j)}=\left\{i \in\{1, \cdots, I\} \mid p_{i}^{(j)}=p^{(j)}\right\}
$$

we obtain:

$$
\begin{aligned}
\sum_{i=1}^{I} p_{i}^{(j)} x_{i}^{(j)}-\max _{i=1, \cdots, I} p_{i}^{(j)} e^{(j)}= & \sum_{i \in \mathcal{I}^{(j)}} p^{(j)} x_{i}^{(j)}+\sum_{i \notin \mathcal{I}^{(j)}} p_{i}^{(j)} x_{i}^{(j)}-p^{(j)} e^{(j)} \\
= & \sum_{i \in \mathcal{I}^{(j)}} p^{(j)} x_{i}^{(j)}+\sum_{i \notin \mathcal{I}^{(j)}} p_{i}^{(j)} x_{i}^{(j)}-p^{(j)} e^{(j)} \\
& +\sum_{i \notin \mathcal{I}^{(j)}} p^{(j)} x_{i}^{(j)}-\sum_{i \notin \mathcal{I}^{(j)}} p^{(j)} x_{i}^{(j)} \\
= & p^{(j)}\left(\sum_{i=1}^{I} x_{i}^{(j)}-e^{(j)}\right) \\
& +\sum_{i \notin \mathcal{I}^{(j)}}\left(p_{i}^{(j)}-p^{(j)}\right) x_{i}^{(j)}
\end{aligned}
$$

The last expression is nonpositive due to (3.5), (3.7), and $p^{(j)}, x_{i}^{(j)} \in \mathbb{R}_{+}, i=$ $1, \cdots, I$.

(ii) Let (3.6) be satisfied. Setting there $p_{i}=p \in \mathbb{R}_{+}^{n}$, we get

$$
\left\langle p, \sum_{i=1}^{I} x_{i}\right\rangle \leqslant\langle p, e\rangle \text { for all } p \in \mathbb{R}_{+}^{n}
$$

Hence, (3.5) is fulfilled.

\section{Theorem 3.3 It holds:}

$$
\begin{aligned}
\min _{p \in \mathbb{R}_{+}^{n}} \operatorname{TLR}(p) & =\min _{p_{1}, \cdots, p_{I} \in \mathbb{R}_{+}^{n}} \operatorname{TLR}\left(p_{1}, \cdots, p_{I}\right) \\
& =\max _{\substack{x_{i} \in X_{i} \\
i=1, \cdots, I}}\left\{\Phi\left(x_{1}, \cdots, x_{I}\right) \mid \sum_{i=1}^{I} x_{i} \leqslant e\right\} .
\end{aligned}
$$

\section{Proof}

$$
\begin{aligned}
& \operatorname{TLR}\left(p_{1}, \cdots, p_{I}\right) \\
= & \max _{\substack{x_{i} \in X_{i} \\
i=1, \cdots, I}}\left[\Phi\left(x_{1}, \cdots, x_{I}\right)-\sum_{i=1}^{I}\left\langle p_{i}, x_{i}\right\rangle+\left\langle\max _{i=1, \cdots, I} p_{i}, e\right\rangle\right] .
\end{aligned}
$$


Using this representation (3.8) of $\operatorname{TLR}\left(p_{1}, \cdots, p_{I}\right)$, we obtain:

$$
\begin{aligned}
& \min _{p_{1}, \cdots, p_{I} \in \mathbb{R}_{+}^{n}} \operatorname{TLR}\left(p_{1}, \cdots, p_{I}\right) \\
& =\min _{p_{1}, \cdots, p_{I} \in \mathbb{R}_{+}^{n}} \max _{\substack{x_{i} \in X_{i} \\
i=1, \cdots, I}}\left[\Phi\left(x_{1}, \cdots, x_{I}\right)-\sum_{i=1}^{I}\left\langle p_{i}, x_{i}\right\rangle+\left\langle\max _{i=1, \cdots, I} p_{i}, e\right\rangle\right] \\
& =\max _{\substack{x_{i} \in X_{i} \\
i=1, \cdots, I}} \Phi\left(x_{1}, \cdots, x_{I}\right)+\min _{p_{1}, \cdots, p_{I} \in \mathbb{R}_{+}^{n}}-\sum_{i=1}^{I}\left\langle p_{i}, x_{i}\right\rangle+\left\langle\max _{i=1, \cdots, I} p_{i}, e\right\rangle \\
& =\max _{\substack{x_{i} \in X_{i} \\
i=1, \cdots, I}}\left\{\begin{array}{l|l}
\left.\mid x_{1}, \cdots, x_{I}\right) & \begin{array}{l}
\sum_{i=1}^{I}\left\langle p_{i}, x_{i}\right\rangle \leqslant\left\langle\max _{i=1, \cdots, I} p_{i}, e\right\rangle \\
\text { for all } p_{i} \in \mathbb{R}_{+}^{n}, i=1, \cdots, I
\end{array}
\end{array}\right\} .
\end{aligned}
$$

Applying Lemma 3.1, the adjoint constraint $\sum_{i=1}^{I} x_{i} \leqslant e$ is equivalent to

$$
\sum_{i=1}^{I}\left\langle p_{i}, x_{i}\right\rangle \leqslant\left\langle\max _{i=1, \cdots, I} p_{i}, e\right\rangle \text { for all } p_{i} \in \mathbb{R}_{+}^{n}, i=1, \cdots, I .
$$

Overall, (A) is the adjoint problem for (PD). Analogously, (A) is the adjoint problem for $(\mathrm{P})$.

Corollary 3.1 Let $\left(p_{i}\right)_{i=1}^{I}$ solve $(P D)$ and $\left(x_{i}\right)_{i=1}^{I}$ solve its adjoint problem $(A)$. Then, the highest offer prices together with consumption bundles

$$
\left(\max _{i=1, \cdots, I} p_{i},\left(x_{i}\right)_{i=1}^{I}\right)
$$

form a Gale equilibrium. Moreover, the ith consumer's bundle $x_{i}^{(j)}$ vanishes if his individual price $p_{i}^{(j)}$ is less than the highest offer price $\max _{i=1, \cdots, I} p_{i}^{(j)}$ for good $j$, i.e.,

$$
x_{i}^{(j)}=0 \text { if } p_{i}^{(j)} \neq \max _{i=1, \cdots, I} p_{i}^{(j)}, \quad i=1, \cdots, I, j=1, \cdots, n
$$

Proof Due to Theorem 3.3:

$$
\begin{aligned}
0 & \leqslant \operatorname{TLR}\left(\max _{i=1, \cdots, I} p_{i}\right)-\Phi\left(x_{1}, \cdots, x_{I}\right) \\
& \stackrel{(3.2)}{\leqslant} \operatorname{TLR}\left(p_{1}, \cdots, p_{I}\right)-\Phi\left(x_{1}, \cdots, x_{I}\right)=0 .
\end{aligned}
$$


Hence, $\max _{i=1, \cdots, I} p_{i}$ solves $(\mathrm{P})$. Due to the fact that (A) is the adjoint problem also for $(\mathrm{P})$,

$$
\left(\max _{i=1, \cdots, I} p_{i},\left(x_{i}\right)_{i=1}^{I}\right)
$$

is a Gale equilibrium according to Definition 2.2.

Further, (3.9) from Theorem 3.3 yields

$$
-\sum_{i=1}^{I}\left\langle p_{i}, x_{i}\right\rangle+\left\langle\max _{i=1, \cdots, I} p_{i}, e\right\rangle=-\sum_{i=1}^{I}\left\langle\max _{i=1, \cdots, I} p_{i}, x_{i}\right\rangle-\left\langle\max _{i=1, \cdots, I} p_{i}, e\right\rangle=0
$$

Thus,

$$
\sum_{i=1}^{I}\left\langle\max _{i=1, \cdots, I} p_{i}-p_{i}, x_{i}\right\rangle=0
$$

or, equivalently,

$$
\left\langle\max _{i=1, \cdots, I} p_{i}-p_{i}^{(j)}, x_{i}^{(j)}\right\rangle=0, \quad i=1, \cdots, I, \quad j=1, \cdots, n .
$$

The latter implies $x_{i}^{(j)}=0$ for $p_{i}^{j} \neq \max _{i=1, \cdots, I} p_{i}^{(j)}$.

\section{Algorithm for Fisher-Gale Equilibrium}

Let us describe how consumers may efficiently adjust their individual prices $\left(p_{i}\right)_{i=1}^{I}$ for approaching the Fisher-Gale equilibrium. This price adjustment corresponds to the quasi-monotone subgradient method [16], which is described in "Appendix" for reader's convenience. It is applied to the minimization of the total logarithmic revenue (PD):

$$
\min _{p_{1}, \cdots, p_{I} \in \mathbb{R}_{+}^{n}} \operatorname{TLR}\left(p_{1}, \cdots, p_{I}\right)
$$

Let $i$ th consumer choose a sequence of positive confidence parameters $\left\{\chi_{i}[t]\right\}_{t} \geqslant 0$, $i=1, \cdots, I$. We consider the following iteration:

\section{Algorithm for Fisher-Gale equilibrium (AFGE)}

Step 1 Consumers determine their current excess supplies $\nabla_{p_{i}} \operatorname{TLR}\left(p_{1}[t], \cdots, p_{I}[t]\right)$ :

(a) $i$ th consumer computes an optimal bundle

$$
x_{i}\left(p_{i}[t]\right) \in \arg \max _{x_{i} \in X_{i}}\left[w_{i} \ln u_{i}\left(x_{i}\right)-\left\langle p_{i}[t], x_{i}\right\rangle\right]
$$


and the corresponding utility prices

$$
q_{i}\left(p_{i}[t]\right)=\frac{w_{i}}{u_{i}\left(x_{i}\left(p_{i}[t]\right)\right)}, \quad i=1, \cdots, I .
$$

(b) $k$ th producer identifies the highest offer prices

$$
p[t]=\max _{i=1, \cdots, I} p_{i}[t]
$$

decides on supply shares

$$
\left(\mu_{i k}[t]\right)_{i=1}^{I} \in M\left(p_{1}[t], \cdots, p_{I}[t]\right),
$$

and supplies to the $i$ th consumer the bundle

$$
\mu_{i k}[t] \circ e_{k}, \quad i=1, \cdots, I .
$$

(c) $i$ th consumer computes his current excess supply

$$
\nabla_{p_{i}} \operatorname{TLR}\left(p_{1}[t], \cdots, p_{I}[t]\right)=\sum_{k}^{K} \mu_{i k}[t] \circ e_{k}-x_{i}\left(p_{i}[t]\right)
$$

Step 2 Consumers accumulate their excess supplies

$$
\begin{aligned}
z_{i}[t] & =z_{i}[t-1]+\nabla_{p_{i}} \operatorname{TLR}\left(p_{1}[t], \cdots, p_{I}[t]\right), \quad z_{i}[-1]=0, \\
i & =1, \cdots, I .
\end{aligned}
$$

Step 3 Consumers compute their price forecasts w.r.t. the confidence parameters $\chi_{i}[t]$

$$
p_{i}^{+}[t]=\frac{\zeta_{i}^{(j)}}{\chi_{i}[t]}\left(-z_{i}[t]\right)_{+}, \quad i=1, \cdots, I,
$$

where $\zeta_{i}^{(j)}$ are positive scaling coefficients.

Step 4 Consumer update

$$
p_{i}[t+1]=\frac{t+1}{t+2} p_{i}[t]+\frac{1}{t+2} p_{i}^{+}[t], \quad i=1, \cdots, I
$$

by combining their previous prices with the forecasts.

First, we give an interpretation for the price forecast (4.3). Recall that $z_{i}[t]$ represents the excess of producers' supply to $i$ th consumer over his demands for good $j$ accumulated up to time $t$. If $z_{i}^{(j)}[t] \geqslant 0$, i.e., supply exceeds demand, then naturally, the long-term forecast is $p_{i}^{+(j)}[t]=0$ for good $j$. In case of $z_{i}^{(j)}[t]<0$, the price forecast $p_{i}^{+(j)}[t]$ is proportional to the accumulated individual excess demand of $i$ th 
consumer with positive scaling coefficients $\zeta_{i}^{(j)}$. Here, $\chi_{i}[t]$ plays the role of a confidence parameter. Namely, $\chi_{i}[t]$ 's express to which extent consumers take into account their excess demands while forecasting prices.

Secondly, let us interpret the price update (4.4):

$$
p_{i}[t+1]=\frac{t+1}{t+2} p_{i}[t]+\frac{1}{t+2} p_{i}^{+}[t] .
$$

Due to the latter, the next price is a convex combination of the previous price and the price forecast. With time advancing, the proportion of the previous price becomes nearly one, but the fraction of the forecast vanishes. Hence, we conclude that our price update corresponds to a behavior of an experienced consumer. He credits his experience much more than the current forecast. Further, from (4.4) we have

$$
p_{i}[t+1]=\frac{1}{t+2}\left(p_{i}[0]+\sum_{r=0}^{t} p_{i}^{+}[r]\right)
$$

The latter means that the prices generated by AFGE can be viewed as historical averages of preceding forecasts. This averaging pattern is also quite natural to assume for consumer's behavior while adjusting prices.

Next, we produce a feasible sequence for the adjoint problem (A) by averaging consumption bundles from AFGE. Along with the prices $\left\{\left(p_{1}[t], \cdots, p_{I}[t]\right)\right\}_{t \geqslant 0}$ generated by algorithm AFGE, we consider the corresponding historical averages of consumption bundles

$$
x_{i}[t] \stackrel{\text { def }}{=} \frac{1}{t+1} \sum_{r=0}^{t} x_{i}(p[r]) \in X_{i}, \quad i=1, \cdots, I,
$$

as well as the corresponding geometric means of utility prices

$$
q_{i}[t] \stackrel{\operatorname{def}}{=}\left(\prod_{r=0}^{t} q_{i}\left(p_{i}[r]\right)\right)^{\frac{1}{t+1}}=\left(\prod_{r=0}^{t} \frac{w_{i}}{u_{i}\left(x_{i}(p[r])\right)}\right)^{\frac{1}{t+1}}, \quad i=1, \cdots, I .
$$

Next Lemma 4.1 estimates the dual gap for the minimization problem (PD) and its adjoint problem (A) evaluated at the historical averages.

For that, we set

$$
\begin{aligned}
& \operatorname{TLR}[t] \stackrel{\text { def }}{=} \operatorname{TLR}\left(p_{1}[t], \cdots, p_{I}[t]\right), \\
& \Phi[t] \stackrel{\text { def }}{=} \Phi\left(x_{1}[t], \cdots, x_{I}[t]\right), \\
& \Phi_{\mathrm{av}}[t] \stackrel{\text { def }}{=} \frac{1}{t+1} \sum_{r=0}^{t} \Phi\left(x_{1}(p[r]), \cdots, x_{I}(p[r])\right), \\
& F[t] \stackrel{\text { def }}{=} \sum_{j=1}^{n}\left(\sum_{i=1}^{I} x_{i}[t]^{(j)}-e\right)_{+}^{2} .
\end{aligned}
$$


TLR $[t]$ is the value of the primal problem (PD), which is computed at the current prices $\left(p_{1}[t], \cdots, p_{I}[t]\right) . \Phi[t]$ is the value of the adjoint problem $(\mathrm{A})$, which is computed at historical averages $\left(x_{1}[t], \cdots, x_{I}[t]\right) . \Phi_{\mathrm{av}}[t]$ is the average value of the adjoint problem (A), which is computed at current consumption bundles $\left(x_{1}(p[r]), \cdots, x_{I}(p[r])\right)$. Note that due to the concavity of $\Phi$ :

$$
\Phi_{\mathrm{av}}[t] \leqslant \Phi[t]
$$

$F[t]$ is the quadratic penalty for violation of the market feasibility constraint:

$$
\sum_{i=1}^{I} x_{i}[t] \leqslant e .
$$

Further, we define the upper and lower remainder terms $b_{t}$ and $d_{t}$ :

$$
\begin{aligned}
& b_{t} \stackrel{\text { def }}{=} \frac{1}{t+1} \sum_{i=1}^{I} \sum_{r=0}^{t} \frac{1}{\chi_{i}[r-1]}, \quad \chi_{i}[-1]=\chi_{i}[0], \\
& d_{t} \stackrel{\text { def }}{=} \frac{\sum_{i=1}^{I} \chi_{i}[t]}{t+1} .
\end{aligned}
$$

Lemma 4.1 Let the sequence $\left\{p_{1}[t], \cdots, p_{I}[t]\right\}_{t \geqslant 0}$ be generated by AFGE with nondecreasing confidence parameters

$$
\chi_{i}[t+1] \geqslant \chi_{i}[t], \quad t \geqslant 0, i=1, \cdots, I .
$$

Then, for all $t \geqslant 0$ it holds:

$$
\begin{aligned}
\operatorname{TLR}[t]-\operatorname{TLR}^{*}-C_{1} d_{t} & \leqslant \operatorname{TLR}[t]-\Phi[t]+\frac{C_{2}}{d_{t}} F[t] \\
& \leqslant \operatorname{TLR}[t]-\Phi_{\mathrm{av}}[t]+\frac{C_{2}}{d_{t}} F[t] \leqslant C_{3} b_{t}
\end{aligned}
$$

with some positive constants $C_{1}, C_{2}, C_{3}>0$.

Proof The proof of Lemma 4.1 is based on the application of the quasi-monotone subgradient method for nonsmooth convex minimization from [16]. Its proof is postponed to "Appendix" for reader's convenience.

In order to arrive at the equilibrium price, consumers need to appropriately adjust their confidence parameters $\left\{\chi_{i}[t]\right\}_{t \geqslant 0}, i=1, \cdots, I$. Next Lemma 4.2 identifies successful adjustment strategies of confidence parameters. Namely, the confidence in the market mechanism increases, but by decreasing increments. This ensures the convergence of the remainder terms $b_{t}, d_{t}$ from Lemma 4.1 . 
Lemma 4.2 Let nondecreasing confidence parameters of the ith consumer satisfy

$$
\chi_{i}[t]-\chi_{i}[t-1] \rightarrow 0, \quad \chi_{i}[t] \rightarrow \infty
$$

Then,

$$
\frac{\chi_{i}[t]}{t+1} \rightarrow 0, \quad \text { and } \frac{1}{t+1} \sum_{r=0}^{t} \frac{1}{\chi_{i}[r-1]} \rightarrow 0
$$

Moreover, the achievable order of convergence in (4.8) is $O\left(\frac{1}{\sqrt{t}}\right)$.

Proof Since $\chi_{i}[t]-\chi_{i}[t-1] \rightarrow 0$, it holds by averaging that $\frac{1}{t+1} \sum_{r=0}^{t}\left(\chi_{i}[r]-\right.$ $\left.\chi_{i}[r-1]\right) \rightarrow 0$. Thus,

$$
\frac{1}{t+1} \chi_{i}[t]=\frac{1}{t+1} \sum_{r=0}^{t}\left(\chi_{i}[r]-\chi_{i}[r-1]\right)+\frac{1}{t+1} \chi_{i}[-1] \rightarrow 0
$$

From $\chi_{i}[t] \rightarrow \infty$ we have $\frac{1}{\chi_{i}[t]} \rightarrow 0$, and also by averaging, $\frac{1}{t+1} \sum_{r=0}^{t} \frac{1}{\chi_{i}[r-1]} \rightarrow$ 0 . The convergence of the order $O\left(\frac{1}{\sqrt{t}}\right)$ can be achieved in (4.8) by choosing $\chi_{i}[t]=$ $O(\sqrt{t})$. In fact, we obtain:

$$
\frac{1}{t+1} \sum_{r=0}^{t} \frac{1}{\chi_{i}[r-1]}=\frac{1}{t+1}\left(\frac{1}{\chi_{i}[-1]}+\frac{1}{\chi_{i}[0]}\right)+\frac{1}{t+1} \sum_{r=1}^{t} \frac{1}{O(\sqrt{r})}
$$

Immediately, we see that $\frac{1}{t+1}\left(\frac{1}{\chi_{i}[-1]}+\frac{1}{\chi_{i}[0]}\right) \rightarrow 0$ as of the order $O\left(\frac{1}{t}\right)$. Note that for a convex univariate function $\xi(r), r \in \mathbb{R}$, and integer bounds $a, b$, we have

$$
\sum_{r=a}^{b} \xi(r) \leqslant \int_{a-1 / 2}^{b+1 / 2} \xi(s) \mathrm{d} s
$$

Hence, we get

$$
\begin{gathered}
\frac{1}{t+1} \sum_{r=1}^{t} \frac{1}{\sqrt{r}} \stackrel{(4.9)}{\leqslant} \frac{1}{t+1} \int_{1-1 / 2}^{t+1 / 2} \frac{1}{\sqrt{s}} \mathrm{~d} s=\left.\frac{2}{t+1} \sqrt{s}\right|_{1 / 2} ^{t+1 / 2} \\
=\frac{2}{t+1}(\sqrt{t+1 / 2}-\sqrt{1 / 2}) \rightarrow 0
\end{gathered}
$$

Here, the order of convergence is $O\left(\frac{1}{\sqrt{t}}\right)$. By assuming $\chi_{i}[t]=O(\sqrt{t})$, the convergence $\frac{\chi_{i}[t]}{t+1}=\frac{\sqrt{t}}{t+1} \rightarrow 0$ is also of the order $O\left(\frac{1}{\sqrt{t}}\right)$. 
Remark 4.1 As in the proof of Lemma 4.2, nondecreasing confidence parameters can be written in the cumulative form:

$$
\chi_{i}[t]=\sum_{r=0}^{t} h_{i}[r]+\chi_{i}[-1]
$$

with incremental confidences $h_{i}[t] \geqslant 0$. Then, the convergence condition (4.7) means that incremental confidences tend to zero and sum up to infinity, i.e.,

$$
h_{i}[t] \rightarrow 0, \quad \sum_{t=0}^{\infty} h_{i}[t]=\infty
$$

The latter coincides with the usual condition imposed on the step sizes of the subgradient method for nonsmooth convex minimization (e.g., [18]). However, in our setting $h_{i}[t]$ play the role of incremental step sizes. This gives rise to suppose that confidence parameters $\chi_{i}[t]$ can be formed by consumers by incremental learning (cf. [33]). In fact, the $i$ th consumer's confidence in the price adjustment process, $\chi_{i}[t]$, increases over time, however, by decreasing increments $h_{i}[t]$. The latter means that consumers properly slow down the pace of their confidence in the market mechanism.

Now, we are ready to prove the main convergence result for AFGE.

Theorem 4.1 Let consumers apply in AFGE confidence parameters satisfying

$$
\chi_{i}[t]-\chi_{i}[t-1] \rightarrow 0, \quad \chi_{i}[t] \rightarrow \infty, \quad i=1, \cdots, I .
$$

Then, the sequence of highest offer prices and historical averages of consumption bundles

$$
\left(\max _{i=1, \cdots, I} p_{i}[t],\left(x_{i}[t]\right)_{i=1}^{I}\right)
$$

from algorithm AFGE, converges to the set of Fisher-Gale equilibria w.r.t. the utility prices

$$
\left(\lim _{t \rightarrow \infty} q_{i}[t]\right)_{i=1}^{I}
$$

The achievable rate of convergence is of the order $O\left(\frac{1}{\sqrt{t}}\right)$.

Proof From Lemma 4.1 we obtain:

$$
\operatorname{TLR}[t]-\operatorname{TLR}^{*}-C_{1} d_{t} \leqslant \operatorname{TLR}[t]-\Phi[t]+\frac{C_{2}}{d_{t}} F[t] \leqslant C_{3} b_{t} .
$$

This inequality is composed by the objective function TLR of the primal problem (PD), computed at the current prices $\left(p_{1}[t], \cdots, p_{I}[t]\right)$, objective function $\Phi$ of its adjoint 
problem (A), computed at historical averages $\left(x_{1}[t], \cdots, x_{I}[t]\right)$, and the quadratic penalty $F[t]$ for violation of the market feasibility constraint:

$$
\sum_{i=1}^{I} x_{i}[t] \leqslant e
$$

Due to the choice of confidence parameters $\chi_{i}[t], i=1, \cdots, I$, Lemma 4.2 provides:

$$
b_{t} \rightarrow 0 \text {, and } d_{t} \rightarrow 0 \text {. }
$$

Using Theorem 3.3, $\left(p_{i}[t]\right)_{i=1}^{I}$ converges toward the solution set of (PD), and $\left(x_{i}[t]\right)_{i=1}^{I}$ converges toward the solution set of (A) by order $O\left(\frac{1}{\sqrt{t}}\right)$. We apply Corollary 3.1 to conclude that the sequence of highest offer prices together with historical averages of consumption bundles

$$
\left(\max _{i=1, \cdots, I} p_{i},\left(x_{i}[t]\right)_{i=1}^{I}\right)
$$

converges to the set of Gale equilibria (cf. Definition 2.2). In order to get the additional convergence to the set of Fisher-Gale equilibria, we apply Theorem 2.1(b). For that, it is enough to show that the sequence of geometric means of utility prices

$$
q_{i}[t] \stackrel{\text { def }}{=}\left(\prod_{r=0}^{t} q_{i}\left(p_{i}[r]\right)\right)^{\frac{1}{t+1}}=\left(\prod_{r=0}^{t} \frac{w_{i}}{u_{i}\left(x_{i}(p[r])\right)}\right)^{\frac{1}{t+1}},
$$

and the sequence of utility prices corresponding to the average consumption

$$
\frac{w_{i}}{u_{i}\left(x_{i}[t]\right)}, \quad i=1, \cdots, I
$$

have the same limit. From Lemma 4.1 we know that $\Phi_{\text {av }}[t]$ and $\Phi[t]$ have the same limit. Recalling the definitions of $\Phi_{\mathrm{av}}[t]$ and $\Phi[t]$, see also (3.4), the sequences

$$
\frac{1}{t+1} \sum_{r=0}^{t} \ln u_{i}\left(x_{i}(p[r])\right) \text { and } \ln u_{i}\left(x_{i}[t]\right), \quad i=1, \cdots, I
$$

have the same limit. Applying exponential and inversion to the latter, the assertion follows.

Open Access This article is distributed under the terms of the Creative Commons Attribution 4.0 International License (http://creativecommons.org/licenses/by/4.0/), which permits unrestricted use, distribution, and reproduction in any medium, provided you give appropriate credit to the original author(s) and the source, provide a link to the Creative Commons license, and indicate if changes were made. 


\section{Appendix}

Appendix is devoted to the proof of Lemma 4.1. For that, we first present the quasimonotone subgradient method for nonsmooth convex minimization from [16]. As already mentioned, the price adjustment AFGE corresponds to this quasi-monotone subgradient method. Using this fact, we prove Lemma 4.1 in the second part of "Appendix."

\section{Quasi-Monotone Subgradient Methods}

We consider the following minimization problem:

$$
\min _{x \in X} f(x)
$$

where $X \subset \mathbb{R}^{n}$ is a closed bounded convex set with nonempty interior int $X$, and $f$ is a convex function on $\mathbb{R}^{n}$. Moreover, let $f$ be representable as a maximum of concave functions, i.e.,

$$
f(x)=\max _{a \in A}[\Phi(a)+\varphi(x, a)],
$$

where $A \subset \mathbb{R}^{m}$ is a closed convex set, $\varphi(\cdot, a)$ is a convex function on $\mathbb{R}^{n}$ for every $a \in A$, and $\Phi, \varphi(x, \cdot)$ are concave functions on $\mathbb{R}^{m}$ for every $x \in X$. Denote by $a(x)$ one of the optimal solutions of the maximization problem in (4.12). Then,

$$
\nabla f(x) \stackrel{\text { def }}{=} \nabla_{x} \varphi(x, a(x))
$$

is a subgradient of $f$ at $x$. Recall that for an arbitrary subgradient $\nabla f(x)$ at $x \in X$ of a convex function $f$ we have

$$
f(y) \geqslant f(x)+\langle\nabla f(x), y-x\rangle, \quad y \in X .
$$

Using the representation (4.12), we also have

$$
\min _{x \in X} f(x)=\min _{x \in X} \max _{a \in A}[\Phi(a)+\varphi(x, a)]=\max _{a \in A}\left[\Phi(a)+\min _{x \in X} \varphi(x, a)\right] .
$$

The latter maximization problem

$$
\max _{a \in A}\left[\Phi(a)+\min _{x \in X} \varphi(x, a)\right]
$$

is called adjoint for (4.11) with the adjoint variable $a \in A$.

For the set $X$, we assume to be known a prox-function $d(x)$.

Definition 4.1 $d: X \mapsto \mathbb{R}$ is called a prox-function for $X$ if the following holds:

- $d(x) \geqslant 0$ for all $x \in X$ and $d(x[0])=0$ for certain $x[0] \in X$; 
- $d$ is strongly convex on $X$ with convexity parameter one:

$$
d(y) \geqslant d(x)+\langle\nabla d(x), y-x\rangle+\frac{1}{2}\|y-x\|^{2}, \quad x, y \in X,
$$

where $\|\cdot\|$ is a norm on $\mathbb{R}^{n}$.

- Auxiliary minimization problem

$$
\min _{x \in X}\{\langle z, x\rangle+\chi d(x)\}
$$

is easily solvable for $z \in \mathbb{R}^{n}, \chi>0$.

As a simple consequence of Definition 4.1, for $x \in X$ we have

$$
d(x) \geqslant d(x[0])+\langle\nabla d(x[0]), x-x[0]\rangle+\frac{1}{2}\|x-x[0]\|^{2} \geqslant \frac{1}{2}\|x-x[0]\|^{2} .
$$
tion:

For a sequence of positive parameters $\{\chi[t]\}_{t \geqslant 0}$, we consider the following itera-

\section{Quasi-monotone Subgradient Method}

Step 1 Take a current subgradient $\nabla f(x[t])=\nabla_{x} \varphi(x[t], a(x[t]))$.

Step 2 Accumulate subgradients $z[t]=z[t-1]+\nabla f(x[t]), z[-1]=0$.

Step 3 Compute the forecast $x^{+}[t]=\arg \min _{x \in X}\{\langle z[t], x\rangle+\chi[t] d(x)\}$.

Step 4 Update by combining $x[t+1]=\frac{t+1}{t+2} x[t]+\frac{1}{t+2} x^{+}[t]$.

Note that from quasi-monotone subgradient method we have

$$
z[t]=\sum_{r=0}^{t} \nabla f(x[r]), \quad x[t+1]=\frac{1}{t+2}\left(x[0]+\sum_{r=0}^{t} x^{+}[r]\right) .
$$

Next Theorem 4.2 is crucial for the convergence analysis of the quasi-monotone subgradient method. It estimates the dual gap for the minimization problem (4.11) and its adjoint problem (4.15) evaluated at the historical averages.

For that, we define the penalty term $h_{t}$ and the remainder term $\rho_{t}, t \geqslant 0$, as follows:

$$
\begin{aligned}
h_{t}(a) & \stackrel{\text { def }}{=} \min _{x \in X}\left\{\varphi(x, a)+\frac{\chi[t]}{t+1} d(x)\right\}, \quad a \in A, \\
\rho_{t} & \stackrel{\text { def }}{=} \frac{1}{t+1} \sum_{r=0}^{t} \frac{1}{2 \chi[r-1]}\|\nabla f(x[r])\|_{*}^{2}, \quad \chi[-1]=\chi[0] .
\end{aligned}
$$


Here, $\|\cdot\|_{*}$ is the conjugate norm to $\|\cdot\|$, i.e.,

$$
\|s\|_{*} \stackrel{\text { def }}{=} \max _{s \in \mathbb{R}^{n}}\{\langle s, x\rangle:\|x\| \leqslant 1\}, \quad s \in \mathbb{R}^{n} .
$$

Note that $\Phi+h_{t}$ is a smoothed version of the objective function in (4.15).

Further, we define the average adjoint state

$$
a[t] \stackrel{\text { def }}{=} \frac{1}{t+1} \sum_{r=0}^{t} a(x[r]), \quad t \geqslant 0 .
$$

Note that $a[t] \in A$, since $A$ is convex. Let us write

$$
\Phi_{\mathrm{av}}[t] \stackrel{\text { def }}{=} \frac{1}{t+1} \sum_{r=0}^{t} \Phi(a(x[r])), \quad t \geqslant 0,
$$

for the average value of the adjoint problem computed along quasi-monotone subgradient method.

Theorem 4.2 is motivated by the estimate sequence technique (e.g., Section 2.2.1 in [18]) and is due to [16]. We decided to present its proof for readers' convenience.

Theorem 4.2 (cf. [16]) Let the sequence $\{x[t]\}_{t \geqslant 0}$ be generated by quasi-monotone subgradient method with nondecreasing parameters

$$
\chi[t+1] \geqslant \chi[t], \quad t \geqslant 0 .
$$

Then, for all $t \geqslant 0$ it holds

$$
f(x[t])-\Phi(a[t])-h_{t}(a[t]) \leqslant f(x[t])-\Phi_{\mathrm{av}}[t]-h_{t}(a[t]) \leqslant \rho_{t} .
$$

Proof We define the average linearization terms $\ell_{t}$ and $\psi_{t}$ for $f$ :

$$
\begin{aligned}
\ell_{t}(x) & \stackrel{\text { def }}{=} \sum_{r=0}^{t} f(x[r])+\langle\nabla f(x[r]), x-x[r]\rangle, \\
\psi_{t} & \stackrel{\text { def }}{=} \min _{x \in X}\left\{\ell_{t}(x)+\chi[t] d(x)\right\} .
\end{aligned}
$$

First, we show by induction that for all $t \geqslant 0$ it holds

$$
f(x[t])-\frac{\psi_{t}}{t+1} \leqslant \rho_{t}
$$

Let us assume that condition (4.22) is valid for some $t \geqslant 0$. Then,

$$
\begin{aligned}
\psi_{t+1} & =\min _{x \in X}\left\{\ell_{t}(x)+f(x[t+1])+\langle\nabla f(x[t+1]), x-x[t+1]\rangle+\chi[t+1] d(x)\right\} \\
& \stackrel{(4.20)}{\geqslant} \min _{x \in X}\left\{\ell_{t}(x)+\chi[t] d(x)+f(x[t+1])+\langle\nabla f(x[t+1]), x-x[t+1]\rangle\right\}
\end{aligned}
$$




$$
\begin{aligned}
& \stackrel{(4.16)}{\geqslant} \min _{x \in X}\left\{\psi_{t}+\frac{1}{2} \chi[t]\left\|x-x^{+}[t]\right\|^{2}+f(x[t+1])+\langle\nabla f(x[t+1]), x-x[t+1]\rangle\right\} \\
& \stackrel{(4.22)}{\geqslant} \min _{x \in X}\left\{\begin{array}{l}
(t+1) f(x[t])-(t+1) \rho_{t} \\
+\frac{1}{2} \chi[t]\left\|x-x^{+}[t]\right\|^{2}+f(x[t+1])+\langle\nabla f(x[t+1]), x-x[t+1]\rangle
\end{array}\right\} \\
& \stackrel{(4.14)}{\geqslant} \min _{x \in X}\left\{\begin{array}{l}
(t+1)[f(x[t+1])+\langle\nabla f(x[t+1]), x[t]-x[t+1]\rangle]-(t+1) \rho_{t} \\
+\frac{1}{2} \chi[t]\left\|x-x^{+}[t]\right\|^{2}+f(x[t+1])+\langle\nabla f(x[t+1]), x-x[t+1]\rangle
\end{array}\right\} .
\end{aligned}
$$

Since $(t+2) x[t+1]=(t+1) x[t]+x^{+}[t]$, we obtain

$$
\begin{aligned}
\psi_{t+1} \geqslant & (t+2) f(x[t+1])-(t+1) \rho_{t} \\
& +\min _{x \in X}\left\{\left\langle\nabla f(x[t+1]), x-x^{+}[t]\right\rangle+\frac{1}{2} \chi[t]\left\|x-x^{+}[t]\right\|^{2}\right\} \\
\geqslant & (t+2) f(x[t+1])-(t+1) \rho_{t}-\frac{1}{2 \chi[t]}\|\nabla f(x[t+1])\|_{*}^{2} \\
= & (t+2) f(x[t+1])-(t+2) \rho_{t+1} .
\end{aligned}
$$

It remains to note that

$$
\psi_{0}=\min _{x \in X}\{f(x[0])+\langle\nabla f(x[0]), x-x[0]\rangle+\chi[0] d(x)\} \stackrel{(4.18)}{\geqslant} f(x[0])-\rho_{0} .
$$

Now, we relate the term $\frac{\psi_{t}}{t+1}$ from (4.22) to the adjoint problem (4.15). It holds due to convexity of $\varphi(\cdot, a), a \in A$ :

$$
\begin{aligned}
& f(x[r])+\langle\nabla f(x[r]), x-x[r]\rangle \\
\stackrel{(4.12),(4.13)}{=} \Phi(a(x[r]))+\varphi(x[r], a(x[r]))+\left\langle\nabla_{x} \varphi(x[r], a(x[r])), x-x[r]\right\rangle & \\
\leqslant & \Phi(a(x[r])+\varphi(x, a(x[r])) .
\end{aligned}
$$

Hence, we obtain due to concavity of $\varphi(x, \cdot), x \in X$ :

$$
\ell_{t}(x) \leqslant \sum_{r=0}^{t} \Phi\left(a(x[r])+\varphi(x, a(x[r])) \leqslant(t+1)\left[\Phi_{\mathrm{av}}[t]+\varphi(x, a[t])\right] .\right.
$$

Finally, we get

$$
\frac{\psi_{t}}{t+1} \leqslant \Phi_{\mathrm{av}}[t]+\min _{x \in X}\left\{\varphi(x, a[t])+\frac{\chi[t]}{t+1} d(x)\right\}=\Phi_{\mathrm{av}}[t]+h_{t}(a[t]) .
$$

Altogether, (4.22) and (4.23) provide the right-hand side of the formula (4.21). The left-hand side is due to

$$
\Phi_{\mathrm{av}}[t]=\frac{1}{t+1} \sum_{r=0}^{t} \Phi(a(x[r])) \leqslant \Phi\left(\frac{1}{t+1} \sum_{r=0}^{t} a(x[r])\right)=\Phi(a[t])
$$

which is a consequence of the concavity of $\Phi$. 
Additionally, we need the following result on the quadratic penalty for general convex optimization problems. From now on, let us consider the maximization problem

$$
\Phi^{*} \stackrel{\text { def }}{=} \max _{a \in A}\left\{\Phi(a) \mid g_{l}(a) \leqslant 0, l=1, \cdots, L\right\},
$$

where $A \subset \mathbb{R}^{m}$ is a closed convex set, $\Phi$ is a concave function, and $g_{l}(\cdot), l=$ $1, \cdots, L$ are convex functions on $\mathbb{R}^{m}$. We assume that the convex feasible set of the maximization problem (4.24) has a Slater point (e.g., [30]). Let $a^{*}$ be an optimal solution of (4.24) with some Lagrange multipliers $\lambda_{l}^{*}, l=1, \cdots, L$, i.e.,

$$
\begin{aligned}
& \left\langle\nabla \Phi\left(a^{*}\right)-\sum_{l=1}^{L} \lambda_{l}^{*} \nabla g_{l}\left(a^{*}\right), a^{*}-a\right\rangle \geqslant 0, \quad \text { for all } a \in A, \\
& \lambda_{l}^{*} \geqslant 0, \quad g_{l}\left(a^{*}\right) \leqslant 0, \quad \sum_{l=1}^{L} \lambda_{l}^{*} g_{l}\left(a^{*}\right)=0 .
\end{aligned}
$$

Lemma 4.3 It holds for $\kappa>0$

$$
\max _{a \in A}\left[\Phi(a)-\frac{\kappa}{2} \sum_{l=1}^{L}\left(g_{l}(a)\right)_{+}^{2}\right] \leqslant \Phi^{*}+\frac{1}{2 \kappa} \sum_{l=1}^{L} \lambda_{l}^{*} .
$$

Proof Due to the concavity of $\Phi$ and the convexity of $g_{l}, l=1, \cdots, L$, it holds for all $a \in A$ :

$$
\begin{aligned}
& \Phi(a) \leqslant \Phi\left(a^{*}\right)+\left\langle\nabla \Phi\left(a^{*}\right), a-a^{*}\right\rangle, \\
& g_{l}(a) \geqslant g_{l}\left(a^{*}\right)+\left\langle\nabla g_{l}\left(a^{*}\right), a-a^{*}\right\rangle .
\end{aligned}
$$

We estimate

$$
\begin{gathered}
\Phi(a) \stackrel{(4.27)}{\leqslant} \Phi\left(a^{*}\right)+\left\langle\nabla \Phi\left(a^{*}\right), a-a^{*}\right\rangle \stackrel{(4.25)}{\leqslant} \Phi^{*}+\sum_{l=1}^{L} \lambda_{l}^{*}\left\langle\nabla g_{l}\left(a^{*}\right), a-a^{*}\right\rangle \\
\stackrel{(4.28)}{\leqslant} \Phi^{*}+\sum_{l=1}^{L} \lambda_{l}^{*}\left(g_{l}(a)-g_{l}\left(a^{*}\right)\right) \stackrel{(4.26)}{=} \Phi^{*}+\sum_{l=1}^{L} \lambda_{l}^{*} g_{l}(a), \quad a \in A .
\end{gathered}
$$

Hence, 


$$
\begin{aligned}
\max _{a \in A}\left[\Phi(a)-\frac{\kappa}{2} \sum_{l=1}^{L}\left(g_{l}(a)\right)_{+}^{2}\right] & \leqslant \Phi^{*}+\max _{a \in A} \sum_{l=1}^{L}\left[\lambda_{l}^{*} g_{l}(a)-\frac{\kappa}{2}\left(g_{l}(a)\right)_{+}^{2}\right] \\
& \leqslant \Phi^{*}+\sum_{l=1}^{L} \max _{b_{l} \in \mathbb{R}} \sum_{l=1}^{L}\left[\lambda_{l}^{*} b_{l}-\frac{\kappa}{2}\left(b_{l}\right)_{+}^{2}\right] \\
& =\Phi^{*}+\sum_{l=1}^{L} \frac{1}{2 \kappa} \lambda_{l}^{*} .
\end{aligned}
$$

\section{Proof of Lemma 4.1}

We start by proving that the price adjustment AFGE is a variant of the quasimonotone subgradient method. For that, it suffices to show that

(1) the price forecast (4.3) can be derived by means of Euclidean prox-functions,

(2) TLR can be represented as the maximum of concave functions.

Firstly, we define the Euclidean prox-functions:

$$
d_{i}(p) \stackrel{\text { def }}{=} \frac{1}{2} \sum_{j=1}^{n} \frac{1}{\zeta_{i}^{(j)}}\left(p^{(j)}\right)^{2}, \quad i=1, \cdots, I,
$$

where $\zeta_{i}^{(j)}$ are positive scaling coefficients. The corresponding norms in Definition 4.1 and their conjugates according to (4.19) are

$$
\|p\|_{i}^{2}=\sum_{j=1}^{n} \frac{1}{\zeta_{i}^{(j)}}\left(p^{(j)}\right)^{2}, \quad\|s\|_{i *}^{2}=\sum_{j=1}^{n} \zeta_{i}^{(j)}\left(s^{(j)}\right)^{2}, \quad i=1, \cdots, I .
$$

For $z_{i}[t] \in \mathbb{R}^{n}, \chi_{i}[t]>0$, we consider the minimization problem as from Step 3 in quasi-monotone subgradient method:

$$
\min _{p_{1}, \cdots, p_{i} \in \mathbb{R}_{+}^{n}}\left\{\sum_{i=1}^{I}\left\langle z_{i}[t], p_{i}\right\rangle+\chi_{i}[t] d_{i}\left(p_{i}\right)\right\} .
$$

Its unique solution is the price forecast (4.3) as from Step 3 in AFGE:

$$
p_{i}^{+(j)}[t]=\frac{\zeta_{i}^{(j)}}{\chi_{i}[t]}\left(-z_{i}^{(j)}[t]\right)_{+}, \quad j=1, \cdots, n, i=1, \cdots, I .
$$

Secondly, it follows from (3.8) that the total logarithmic revenue is representable as a maximum of concave functions:

$$
\operatorname{TLR}\left(p_{1}, \cdots, p_{I}\right)=\max _{\substack{x_{i} \in X_{i} \\ i=1, \cdots, I}} \Phi\left(x_{1}, \cdots, x_{I}\right)+\varphi\left(p_{1}, \cdots, p_{K}, x_{1}, \cdots, x_{I}\right)
$$


where

$$
\varphi\left(p_{1}, \cdots, p_{I}, x_{1}, \cdots, x_{i}\right)=-\sum_{i=1}^{I}\left\langle p_{i}, x_{i}\right\rangle+\left\langle\max _{i=1, \cdots, I} p_{i}, e\right\rangle .
$$

Overall, we may apply Theorem 4.2 to get the following inequality:

$$
\operatorname{TLR}\left(p_{1}[t], \cdots, p_{I}[t]\right)-\Phi_{\mathrm{av}}[t]-h_{t}\left(x_{1}[t], \cdots, x_{I}[t]\right) \leqslant \rho_{t},
$$

where

$$
\begin{aligned}
& h_{t}\left(x_{1}[t], \cdots, x_{I}[t]\right) \\
= & \min _{p_{1}, \cdots, p_{I} \in \mathbb{R}_{+}^{n}}\left\{\varphi\left(p_{1}, \cdots, p_{I}, x_{1}[t], \cdots, x_{I}[t]\right)+\frac{1}{t+1} \sum_{i=1}^{I} \chi_{i}[t] d_{i}\left(p_{i}\right)\right\}, \\
\rho_{t}= & \frac{1}{t+1} \sum_{i=1}^{I} \sum_{r=0}^{t} \frac{1}{2 \chi_{i}[r-1]}\left\|\nabla_{p_{i}} \operatorname{TLR}\left(p_{1}[t], \cdots, p_{I}[t]\right)\right\|_{i *}^{2} .
\end{aligned}
$$

We relate the penalty term $h_{t}$ to $F[t]$ from Lemma 4.1. For that, we define the Euclidean prox-function

$$
d(p) \stackrel{\text { def }}{=} \frac{1}{2} \sum_{j=1}^{n}\left(p^{(j)}\right)^{2}
$$

It holds:

$$
\begin{aligned}
& h_{t}\left(x_{1}[t], \cdots, x_{I}[t]\right) \\
\leqslant & \min _{p \in \mathbb{R}_{+}^{n}}\left\{\varphi\left(p, \cdots, p, x_{1}[t], \cdots, x_{I}[t]\right)+\frac{1}{t+1} \sum_{i=1}^{I} \chi_{i}[t] d_{i}(p)\right\} \\
= & \min _{p \in \mathbb{R}_{+}^{n}}\left\{\left\langle p, e-\sum_{i=1}^{I} x_{i}[t]\right\rangle+\frac{1}{t+1} \sum_{i=1}^{I} \chi_{i}[t] d_{i}(p)\right\} \\
\leqslant & \min _{p \in \mathbb{R}_{+}^{n}}\left\{\left\langle p, e-\sum_{i=1}^{I} x_{i}[t]\right\rangle+\frac{\sum_{i=1}^{I} \chi_{i}[t]}{t+1} \frac{1}{\min _{i, j} \zeta_{i}^{(j)}} d(p)\right\} \\
= & -\frac{t+1}{\sum_{i=1}^{I} \chi_{i}[t]} \min _{i, j} \frac{\zeta_{i}^{(j)}}{2} \sum_{j=1}^{n}\left(\sum_{i=1}^{I} x_{i}^{(j)}[t]-e\right)_{+} \\
= & -\frac{C_{2}}{d_{t}} F[t],
\end{aligned}
$$

where $C_{2}=\min _{i, j} \frac{\zeta_{i}^{(j)}}{2}$ 
Now, we relate the remainder term $\rho_{t}$ to $b_{t}$ from Lemma 4.1. For that, let the constant $C_{3}>0$ bound the sequence of $i$ th consumer's excess supplies:

$$
\left\|\nabla_{p_{i}} \operatorname{TLR}\left(p_{1}[t], \cdots, p_{I}[t]\right)\right\|_{i *}^{2} \leqslant 2 C_{3}, \quad t \geqslant 0, i=1, \cdots, I,
$$

where, due to (4.1),

$$
\nabla_{p_{i}} \operatorname{TLR}\left(p_{1}[t], \cdots, p_{I}[t]\right)=\sum_{k}^{K} \mu_{i k}[t] \circ e_{k}-x_{i}\left(p_{i}[t]\right) .
$$

The existence of $C_{3}$ in (4.31) follows from the compactness of the consumption sets $X_{i}, i=1, \cdots, I$ (see Sect. 2). Then, it holds

$$
\rho_{t}=\frac{1}{t+1} \sum_{i=1}^{I} \sum_{r=0}^{t} \frac{1}{2 \chi_{i}[r-1]}\left\|\nabla_{p_{i}} \operatorname{TLR}\left(p_{1}[t], \cdots, p_{I}[t]\right)\right\|_{i *}^{2} \leqslant C_{3} b_{t} .
$$

Altogether, we estimated

$$
h_{t}\left(x_{1}[t], \cdots, x_{I}[t]\right) \leqslant-\frac{C_{2}}{d_{t}} F[t], \quad \rho_{t} \leqslant C_{3} b_{t} .
$$

Substituting this into (4.30), we get the right-hand side of (4.6) in Lemma 4.1:

$$
\operatorname{TLR}[t]-\Phi_{\mathrm{av}}[t]+\frac{C_{2}}{d_{t}} F[t] \leqslant C_{3} b_{t}
$$

Now, we estimate the dual gap in Lemma 4.1 from below. For that, we apply Lemma 4.3 and Theorem 3.3 to obtain

$$
\begin{aligned}
\Phi[t]-\frac{C_{2}}{d_{t}} F[t] & =\Phi\left(x_{1}[t], \cdots, x_{I}[t]\right)-\frac{C_{2}}{d_{t}} \sum_{j=1}^{n}\left(\sum_{i=1}^{I} x_{i}^{(j)}[t]-e\right)_{+}^{2} \\
& \leqslant \max _{\substack{x_{i} \in X_{i} \\
i=1, \cdots, I}} \Phi\left(x_{1}, \cdots, x_{I}\right)-\frac{C_{2}}{d_{t}} \sum_{j=1}^{n}\left(\sum_{i=1}^{I} x_{i}^{(j)}-e\right)_{+}^{2} \\
& \leqslant \max _{\substack{x_{i} \in X_{i}, i=1, \cdots, I \\
\sum_{i=1}^{I} x_{i} \leqslant e}} \Phi\left(x_{1}, \cdots, x_{I}\right)+\frac{d_{t}}{4 C_{2}} \sum_{j=1}^{n} p^{*(j)}=\mathrm{TLR}^{*}+C_{1} d_{t},
\end{aligned}
$$

where $C_{1}=\frac{\sum_{j=1}^{n} p^{*(j)}}{4 C_{2}}$ and $p^{*}$ is an equilibrium price. The latter exists due to the assumption on market productivity. Note that Lagrange multipliers for the market feasibility constraint in the adjoint problem (A) coincide with minimizers $p^{*}$ of the total logarithmic revenue TLR. 
Finally, we estimate

$$
\operatorname{TLR}[t]-\Phi[t]+\frac{C_{2}}{d_{t}} F[t] \geqslant \operatorname{TLR}[t]-\operatorname{TLR}^{*}-C_{1} d_{t}
$$

This is the left-hand side of (4.6) in Lemma 4.1. It remains to note that the inequality in the middle of (4.6) follows due to the concavity of $\Phi$ :

$$
\Phi_{\mathrm{av}}[t] \leqslant \Phi[t]
$$

\section{References}

[1] Brainard, W.C., Scarf, H.: How to compute equilibrium prices in 1891. Am. J. Econ. Sociol. 64(1), 57-83 (2005)

[2] Eisenberg, E., Gale, D.: Consensus of subjective probabilities: the Pari-Mutuel method. Ann. Math. Stat. 30(1), 165-168 (1959)

[3] Eisenberg, E.: Aggregation of utility functions. Manag. Sci. 7(4), 337-350 (1961)

[4] Jain, K., Vazirani, V., Ye., Y.: Market equilibria for homothetic, quasi-concave utilities and economies of scale in production. In: Proceeding SODA'05, pp. 63-71 (2005)

[5] Chen, L., Ye, Y., Zhang, J.: A note on equilibrium pricing as convex optimization. In: Internet and Network Economics, Lecture Notes in Computer Science, vol. 4858, pp. 7-16 (2007)

[6] Gale, D.: The Theory of Linear Economic Models. McGraw Hill, New York (1960)

[7] Devanur, N.R., Papadimitriou, Ch.H., Saberi, A., Vazirani, V.V.: Market equilibrium via a primal-dual algorithm for a convex program. J. ACM 55(5), 1-18 (2008)

[8] Ye, Y.: A path to the Arrow-Debreu competitive market equilibrium. Math. Program. 111(1-2), 315-348 (2008)

[9] Codenotti, B., Mccune, B., Varadarajan, V.: Market equilibrium via the excess demand function. In: Proceedings of the 37th Annual ACM Symposium on Theory of Computing, pp. 74-83. ACM Press, New York (2005). https://doi.org/10.1145/1060590.1060601

[10] Garg, R., Kapoor, S.: Auction algorithms for market equilibrium. Math. Oper. Res. 31(4), 714-729 (2006)

[11] Birnbaum, B., Devanur, N., Xiao, L.: Distributed algorithms via gradient descent for fisher markets. In: ACM Conference on Electronic Commerce, pp. 127-136 (2011)

[12] Fleischer, L., Garg, R., Kapoor, S., Khandekar, R., Saberi, A.: A fast and simple algorithm for computing market equilibria. In: Internet and Network Economics, Lecture Notes in Computer Science, vol. 5385, pp. 19-30 (2008)

[13] Milgrom, P.: Putting auction theory to work: the simultaneous ascending auction. J. Polit. Econ. 108(2), 245-272 (2000)

[14] Codenotti, B., Pemmaraju, S., Varadarajan, V.: The computation of market equilibria. Acm Sigact News 35(4), 23-37 (2004)

[15] Vazirani, V.: Combinatorial algorithms for market equilibria. In: Nisan, N., Roughgarden, T., Tardos, E., Vazirani, V.V. (eds.) Algorithmic Game Theory. Cambridge University Press, Cambridge (2007)

[16] Nesterov, Yu., Shikhman, V.: Quasi-monotone subgradient methods for nonsmooth convex minimization. J. Optim. Theory Appl. 165(3), 917-940 (2015)

[17] Cheung, Y.K., Cole, R., Devanur, N.: Tatonnement beyond gross substitutes? Gradient descent to the rescue. STOC 13, 191-200 (2013)

[18] Nesterov, Yu.: Introductory Lectures on Convex Optimization. Kluwer, Boston (2004)

[19] Mas-Colell, A., Whinston, M.D., Green, J.R.: Microeconomic Theory. Oxford University Press, New York (1995)

[20] Rubinstein, A.: Equilibrium in the jungle. Econ. J. 117(522), 883-896 (2007)

[21] Schotter, A.: Microeconomics: a modern approach. South-Western Cengage Learning, Mason (2009)

[22] Mont, O.: Consumption and ecological economics: towards sustainability. In: Pertsova, C.C. (ed.) Ecological Economics Research Trends, pp. 13-44. Nova Sciences Publishers, Hauppauge, NY (2007)

[23] Marschak, T., Selten, R.: General Equilibrium with Price-Making Firms. Springer, Berlin (1974) 
[24] Negishi, T.: Welfare economics and existence of an equilibrium for a competitive economy. Metroeconomica 12(2-3), 92-97 (1960)

[25] Mantel, R.R.: The welfare adjustment process: its stability properties. Int. Econ. Rev. 12(3), 415-439 (1971)

[26] Mullainathan, S., Thaler, R.H.: Behavioral economics. In: International Encyclopedia of Social and Behavioral Sciences, Smelser, N.J., Baltes, P.B. (Editors-in-Chief), pp. 1094-1100. Elsevier, Amsterdam (2001)

[27] Cherchye, L., Demuynck, T., De Rock, B.: Is utility transferable? A revealed preference analysis. Theor. Econ. 10(1), 51-65 (2015)

[28] Miyake, M.: On the applicability of Marshallian partial-equilibrium analysis. Math. Soc. Sci. 52(2), 176-196 (2006)

[29] Vives, X.: Small income effects: a Marshallian theory of consumer surplus and downward sloping demand curves. Rev. Econ. Stud. 54, 87-103 (1987)

[30] Rockafellar, R.T.: Convex Analysis. Princeton University Press, Princeton (1970)

[31] Zălinescu, C.: Convex Analysis in General Vector Spaces. World Scientific, Singapore (2002)

[32] Rosen, J.B.: Existence and uniqueness of equilibrium points for concave $n$-person games. Econometrica 31(3), 520-534 (1965)

[33] Seel, N.M. (ed.): Incremental learning. In: Encyclopedia of the Sciences of Learning, Springer, Berlin (2012) 